\title{
Focal Liver Lesions
}

\author{
Wolfgang Schima, Dow-Mu Koh, and Richard Baron ${ }^{\dagger}$
}

\section{Learning Objectives}

- To learn the optimal imaging techniques and the relevance of differential diagnosis for liver diseases

- To discuss current indications for liver-specific contrast agents

- To review the imaging features of benign and malignant focal liver lesions

- To discuss the differential diagnosis of primary and secondary hepatic tumors

\subsection{Introduction}

Multidetector computed tomography (MDCT) and magnetic resonance (MR) imaging provide noninvasive insights into liver anatomy and the pathophysiology of liver diseases, which allows for better disease diagnosis, monitoring of disease evolution and treatment response, as well as for guiding treatment decisions. Understanding the application of different imaging techniques is critical for the management of focal liver lesions. In the current climate of challenging health economics, the most appropriate and cost effective modality should always be utilized. For liver imaging, ultrasonography (US) is widely available, noninvasive,

\footnotetext{
${ }^{\dagger}$ Author was deceased

W. Schima, M.D., M.Sc. ( $ه)$

Department of Radiology, Göttlicher Heiland Krankenhaus, Barmherzige Schwestern Krankenhaus, Sankt Josef Krankenhaus, Vinzenzgruppe, Vienna, Austria

e-mail: wolfgang.schima@khgh.at

D.-M. Koh

Department of Radiology, Royal Marsden Hospital, Sutton, UK
}

and often used in the community for disease screening but has unfortunately limited diagnostic sensitivity and specificity. Contrast-enhanced MDCT remains the modality of choice for routine liver imaging. MR imaging is still used largely as a problem-solving tool when MDCT or US is equivocal or if there is concern for malignancy in high-risk populations.

In this chapter, we will highlight imaging of focal liver lesions, focusing on the use of MDCT and MR imaging for disease detection and characterization. The reader should learn how to optimize CT and MR imaging in his/her own practice, understand how to apply and interpret CT and MR imaging for the management of focal liver lesions, and appreciate the expanding role of liver-specific MR contrast agents for lesion characterization.

\subsection{MDCT Imaging Techniques}

MDCT allows imaging to be performed in multiple planes. Using a 64-plus-detector-row system, the entire liver can be scanned within 1-4 s using a submillimeter detector configuration allowing for high-quality multiplanar reconstructions (MPR) [1]. When viewed axially, reconstructed sections of $2.5-3 \mathrm{~mm}$ thickness with an overlap of $0.5-1 \mathrm{~mm}$ are usually used in clinical practice. Thinner slices do not improve lesion conspicuity because of increased image noise [2] that can decrease diagnostic specificity [3]. The amount of contrast material administered can be calculated by a patient's weight, but $0.5 \mathrm{~g}$ iodine/kg b.w. is typical (i.e., $1.7 \mathrm{~mL} / \mathrm{kg}$ b.w. at $300 \mathrm{mg} /$ $\mathrm{mL}$ ). The total amount of iodine administered determines the quality of the portal venous imaging phase, with the aim of increasing the liver attenuation by $50 \mathrm{HU}$ after contrast injection [4]. To achieve good arterial phase imaging, a relatively high contrast medium injection rate of $4-5 \mathrm{~mL} / \mathrm{s}$ is recommended [5]. On the other hand, studies have shown that a fixed injection duration of $30 \mathrm{~s}$ (meaning that the injection rate will differ 
according to patient's weight) also provides consistent image quality.

The timing of the image acquisition in relation to contrast media administration depends on whether imaging is required during early arterial phase (for arterial anatomy only), late arterial phase (for hypervascular tumor detection and characterization), or venous phase (for follow-up imaging and hypovascular tumor detection). For the detection and characterization of focal liver lesions, late arterial phase imaging (with a delay of aortic transit time plus 15-18 s) [6, 7] and a venous phase scan (20-30 s interscan delay or with fixed delay of $\sim 60-70 \mathrm{~s}$ ) are performed. However, the use of combinations of these imaging phases also depends on specific indications [8]. Automated methods of measuring arterial enhancement (aortic transit time) on CT, often termed bolus tracking, have replaced the use of fixed scan-delay times because it provides better coincidence of scanning with peak enhancement of liver tumors (in the late arterial phase) and the liver parenchyma (in the venous phase).

Different techniques for dose reduction and optimization of image quality are now widely in use: automatic exposure control by tube current ( $\mathrm{mA}$ ) modulation, selection of lower tube potential $(\mathrm{kVp})$, and adaptive dose shielding to minimize overscanning in the $\mathrm{z}$-axis, to name a few. Conventional filtered back projection (FBP), the standard CT image reconstruction technique for many years, has given way to iterative reconstruction (IR). IR uses loop-wise raw data correction to reduce image noise, thus allowing imaging to be performed at reduced $\mathrm{kVp}$ or $\mathrm{mAs}$, with lower radiation dose but comparable image quality. All major manufactur- ers now provide iterative reconstruction techniques (SAFIRE, ADMIRE, Siemens; iDose, IMR, Philips; ASIR, MBIR, GE Healthcare; AIDR, AIDR 3D, Toshiba) [9]. Stepwise IR reduces CT noise levels. However, high levels of IR may induce a pixelated (plastic-like) image texture and may render image quality unacceptable [10]. A substantial dose reduction of $38-55 \%$ is possible with IR without compromising image quality [11-13] (Fig. 17.1). In recent years, dual-energy and spectral CT technique has emerged, where the utilization of dual-source or polychromatic X-ray beams and the differential attenuation of such beams of different energies in tissues are applied to improve the detection of hypervascular hepatocellular carcinomas [14] or for the quantification of hepatic iron content [15]. However, dual-energy CT technology is still not widely employed in clinical practice despite potential merits, in part because of the post-processing time required to generate the appropriate images.

\subsection{MR Imaging Technique}

MR imaging of the liver can now be performed at both 1.5 and $3.0 \mathrm{~T}$; the latter has significantly improved in image quality due to advancements in both imaging hardware and software. MR examination of the liver should include unenhanced T1-weighted and T2-weighted sequences, as well as contrast-enhanced sequences. Specific acquisition sequences vary by manufacturer, patient compliance, and the clinical question being addressed.
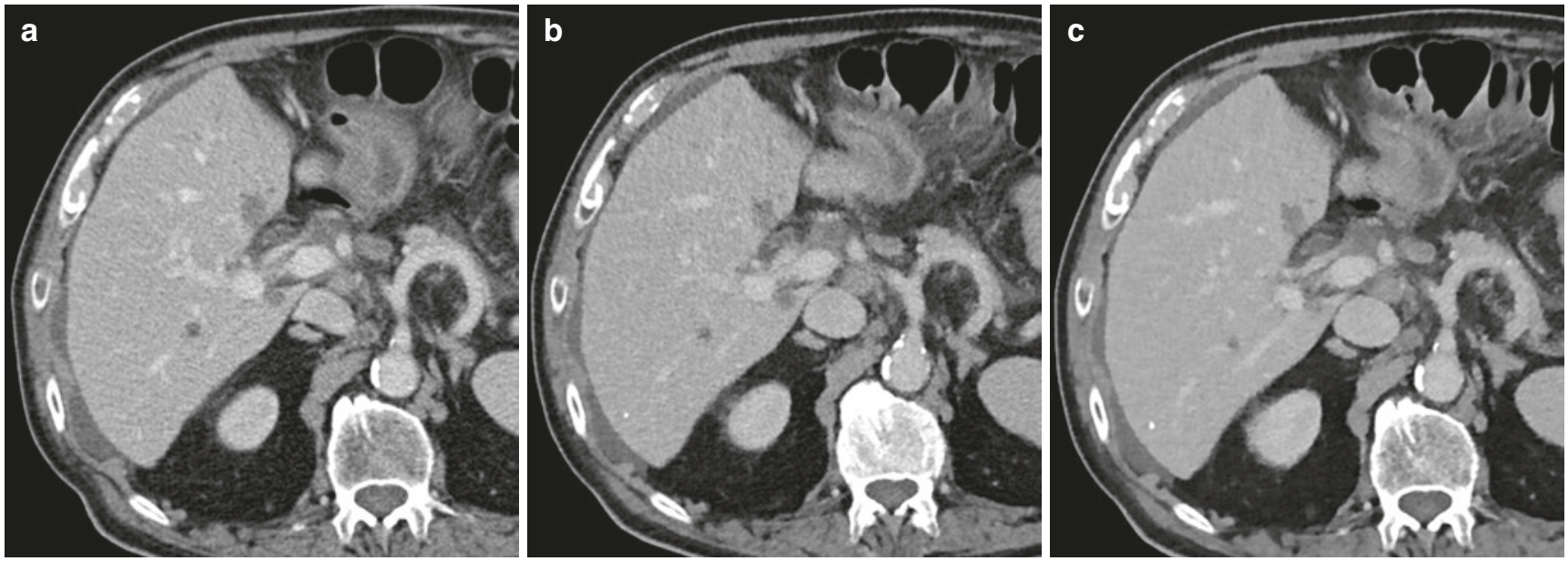

Fig. 17.1 Dose reduction using iterative reconstruction techniques at MDCT. (a) Normal dose MDCT in the venous phase $(120 \mathrm{kVp}$, ref. mAs 230) reconstructed with standard filtered back projection shows colorectal liver metastases. (b) Image appearance $(120 \mathrm{kVp}$, ref. mAs 150) using iterative reconstruction (SAFIRE level 3 ) is slightly differ- ent in general, due to reduced image noise. The lesions are shown with the same conspicuity. Patient dose is reduced by 36\%. (c) At higher iterative reconstruction levels (SAFIRE level 5), the image appearance is pixelated ("plastic-like"), especially seen at the liver parenchyma and the perirenal fat 
In- and opposed-phase (or out-of-phase) T1-weighted imaging is recommended for maximal tumor detection and for characterization of fat containing tumors and the presence of steatosis. T1-weighted MRI can be now performed using a 3D DIXON technique, which can generate in-phase, out-of-phase, water-only, and fat-only images of the whole liver volume in a single breath-hold acquisition. The resultant water-only images have been shown to improve the uniformity of fat suppression at $3 \mathrm{~T}$, compared with conventional spectral fat suppression technique [16]. The use of the DIXON images for dynamic contrast-enhanced acquisition has also been shown to improve the detection of hepatocellular carcinoma compared with standard fat-suppressed sequences.

Another useful recent implementation is non-Cartesian radial T1-weighted imaging, which allows 3D volume T1-weighted imaging of the liver to be performed in free breathing. This allows good quality T1-weighted of the liver to be obtained in patients with poor breath holding (e.g., elderly, breathless adults, or young children) (Fig. 17.2), especially during dynamic contrast-enhanced acquisitions [17]. T2-weighted pulse sequences with fat suppression provide better lesion contrast than nonfat-suppressed sequences and are also widely used.

Diffusion-weighted imaging (DWI) has become a standard technique in liver imaging, and it is now available on all scanners. In general, DWI depends upon the microscopic mobility of water, called Brownian motion, in tissue. Watermolecule diffusion (and thus the measured signal intensity) depends on tissue cellularity, tissue organization, integrity of cellular membranes, and extracellular space tortuosity. Usually, lower water diffusion is found in most solid tumors,

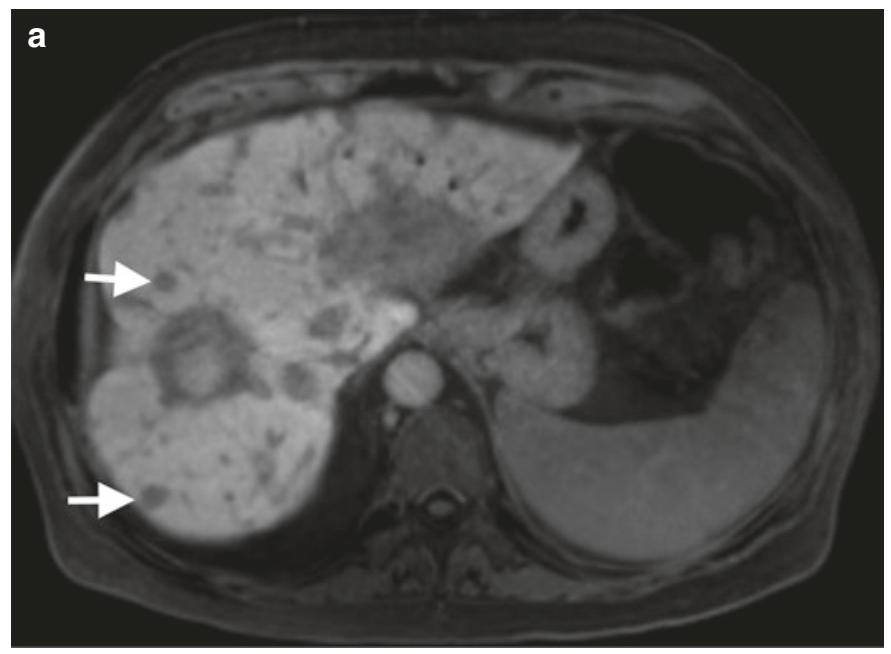

Fig. 17.2 Radial acquisition technique. Colorectal liver metastases. Hepatobiliary phase imaging performed at 20 min after gadoxetate contrast administration using (a) free breathing radial acquisition fatsuppressed gradient echo and (b) breath-hold volume interpolated which are attributed to their high cellularity [18]. Thus, DWI is helpful for detecting liver solid focal liver lesions [19-21]. By performing diffusion-weighted imaging using two or more b-values, we can quantify the apparent diffusion coefficient (ADC) of liver tissues. Benign focal liver lesions have been shown to have higher ADC value than malignant liver lesions, although there is significant overlap [22]. Nonetheless, quantitative ADC values may be useful to support lesion characterization and for identifying early tumor response to treatment, which is currently being investigated.

Imaging after the administration of intravenous contrast agents remains the cornerstone for liver MR imaging. Of these, nonspecific extracellular gadolinium contrast medium is still most widely used. Following the intravenous (IV) bolus injection of extracellular gadolinium-based contrast agents, dynamic imaging (using volumetric T1-weigthed imaging) is performed in characterizing lesion, detecting lesion, evaluating tumor response to therapy, and detecting marginal recurrences after tumor ablation.

Liver-specific (or hepatobiliary) MR contrast agents are available and have specific roles in the management of focal liver lesions. These include gadobenate dimeglumine (MultiHance $^{\circledR}$, Bracco) and gadoxetic acid (Primovist ${ }^{\circledR}$ or Eovist $^{\circledR}$, Bayer Healthcare). Liver-specific MR contrast agents are also usually administered IV as a bolus, as with nonspecific gadolinium chelates for dynamic imaging. However, imaging is also performed at a delayed liverspecific or hepatobiliary phase, the timing of this differs according to the contrast agent. These liver-specific agents are taken up into hepatocytes to varying extent (gadobenate dimeglumine 4-5\%; gadoxetic acid $~ 50 \%$ ), resulting in avid $\mathrm{T} 1$ enhancement of the liver parenchyma in the hepatobiliary

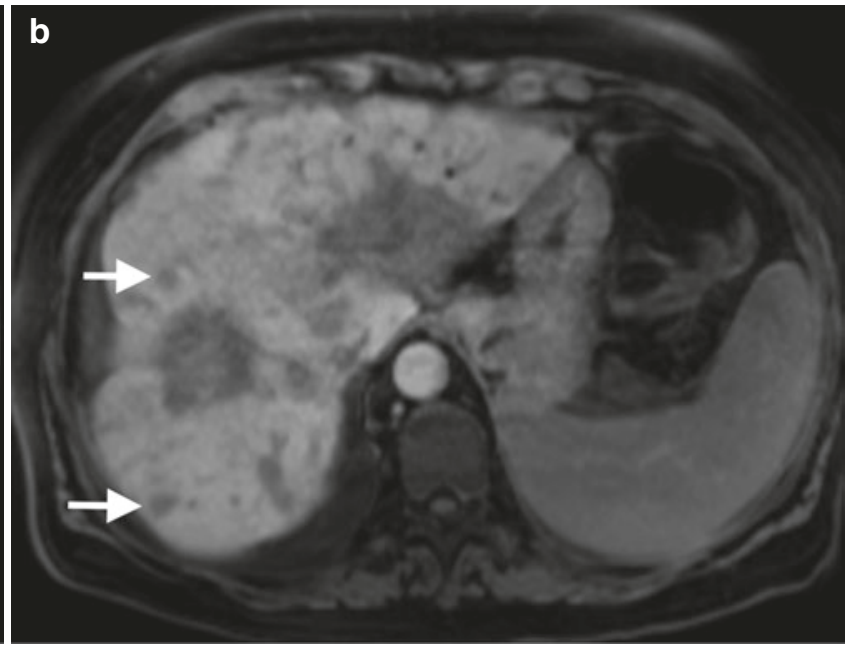

fat-suppressed gradient echo technique. Note that the free-breathing acquisition in this patient resulted in better delineation of the smaller liver metastases as T1 hypointense lesions against the enhancing liver parenchyma (arrows) 
phase, which is performed at 20 min for gadoxetic acid and about $1-2 \mathrm{~h}$ for gadobenate dimeglumine after contrast administration. Liver-specific contrast agents have been shown to improve the detection of liver metastases [23-26], especially when used in combination with diffusion-weighted MR imaging.

\subsection{Benign Hepatic Lesions}

\subsubsection{Cysts}

Simple hepatic cysts are common, occurring in 5-14\% of the general population. As they are usually asymptomatic, they are detected incidentally on US, CT, or MR imaging. On CT, hepatic cysts are well circumscribed and typically show attenuation values similar to water $(0-15 \mathrm{HU})$, although smaller cysts may show higher attenuation values due to partial volume effects. Cysts should not show mural thickening, nodularity, or contrast enhancement. Small cysts $(\leq 3 \mathrm{~mm}$ in size) may pose a diagnostic challenge in the cancer patient on CT as they are too small to fully characterize and stability on follow-up imaging is important to reassure. Nonetheless, the majority of small hypodense liver lesions even in the oncology patient are usually benign.

On MR imaging examinations, cysts are well-defined, homogeneous lesions that appear hypointense on T1-weighted images and markedly hyperintense on T2-weighted images. Their marked hyperintensity on T2-weighted imaging provides greater confidence toward the diagnosis of small cysts on MRI.

\subsubsection{Hemangioma}

Hemangioma is the most common benign liver tumor. On US, liver hemangioma appears circumscribed, well-defined, hyperechoic, and associated with distal acoustic enhancement. Small hemangiomas usually appear homogeneous, but larger hemangiomas $(>4 \mathrm{~cm})$ can show a heterogeneous appearance.

On CT, hemangiomas are well-defined hypodense masses. They are hypointense on T1-weighted and markedly hyperintense on T2-weighted imaging, sometimes with a lobular contour. Hyperintensity on T2-weighted MRI helps to differentiate hemangiomas from other solid neoplasms [27, 28]. At a relatively long T2 echo time (140 ms or longer), a homogeneously bright lesion is characteristic of a benign lesion, such as a cyst or hemangioma. Exceptions include cystic or mucinous metastases, gastrointestinal stromal tumor (GIST), and neuroendocrine tumor metastases.
Hemangiomas show three distinctive patterns of enhancement at CT/MRI (type I to III) [29], where there is characteristically enhancement that closely follows the enhancement of blood pool elsewhere [30]. Small lesions (up to $\sim 2 \mathrm{~cm}$ ) may show immediate and complete enhancement in the arterial phase, with sustained enhancement in the venous and delayed phases (type I, "flash filling") [31] (Fig. 17.3). On delayed imaging, the enhancement usually fades to a similar extent as the blood pool. The most common enhancement pattern is peripheral nodular discontinuous enhancement, which progressively fill-in over time (type II). Larger lesions $(>5 \mathrm{~cm}$ ) or lesions with central thrombosis/fibrosis may lack central fill-in (type III) (Fig. 17.4). When evaluated using liver-specific contrast agents, the appearance of hemangiomas in the dynamic arterial and venous phases is similar to that with nonspecific gadolinium chelates. However, in the delayed phase, after $3 \mathrm{~min}$, there may be "pseudowashout" (hypointensity) due to early hepatocellular enhancement of liver parenchyma (Fig. 17.5). In the hepatobiliary phase, hemangiomas may appear hypointense to the parenchyma, thus mimicking liver metastases. In this instance, DWI may help to differentiate between hemangioma and other solid lesions, as the apparent diffusion coefficient (ADC) of uncomplicated hemangiomas is significantly higher (typically $>1.70 \times 10^{-3} \mathrm{~s} / \mathrm{mm}^{2}$ ) than in malignant solid lesions $[22,32]$.

\subsubsection{Focal Nodular Hyperplasia}

Focal nodular hyperplasia (FNH) is a benign lesion that can cause confusion when incidentally detected during abdominal imaging. On ultrasound, the lesion is usually isoechoic or slightly hypoechoic [33] to liver, but appears hypoechoic in patients with diffuse hepatic steatosis. Typically, FNH demonstrates a lobular contour, which is uncommon in malignant lesions. A central scar is present in about $67 \%$ of larger lesions and about $33 \%$ of smaller lesions [34]. The central scar in FNH is usually hyperintense on T2-weighted images, with a comma-shaped or spoke-wheel appearance, which can be distinguished from fibrolamellar HCC, where the central scar, when present, is predominately low signal intensity on T2-weighted MR. Color/power Doppler US may show blood flow within the scar [35].

FNH is isodense or minimally hypodense on unenhanced and equilibrium-phase post-contrast CT and may be only suspected because of the presence of mass effect on adjacent vessels. On unenhanced T1- and T2-weighted MR images, FNH returns signal intensity similar to hepatic parenchyma but is usually slightly different on either T1- or T2-weighted 

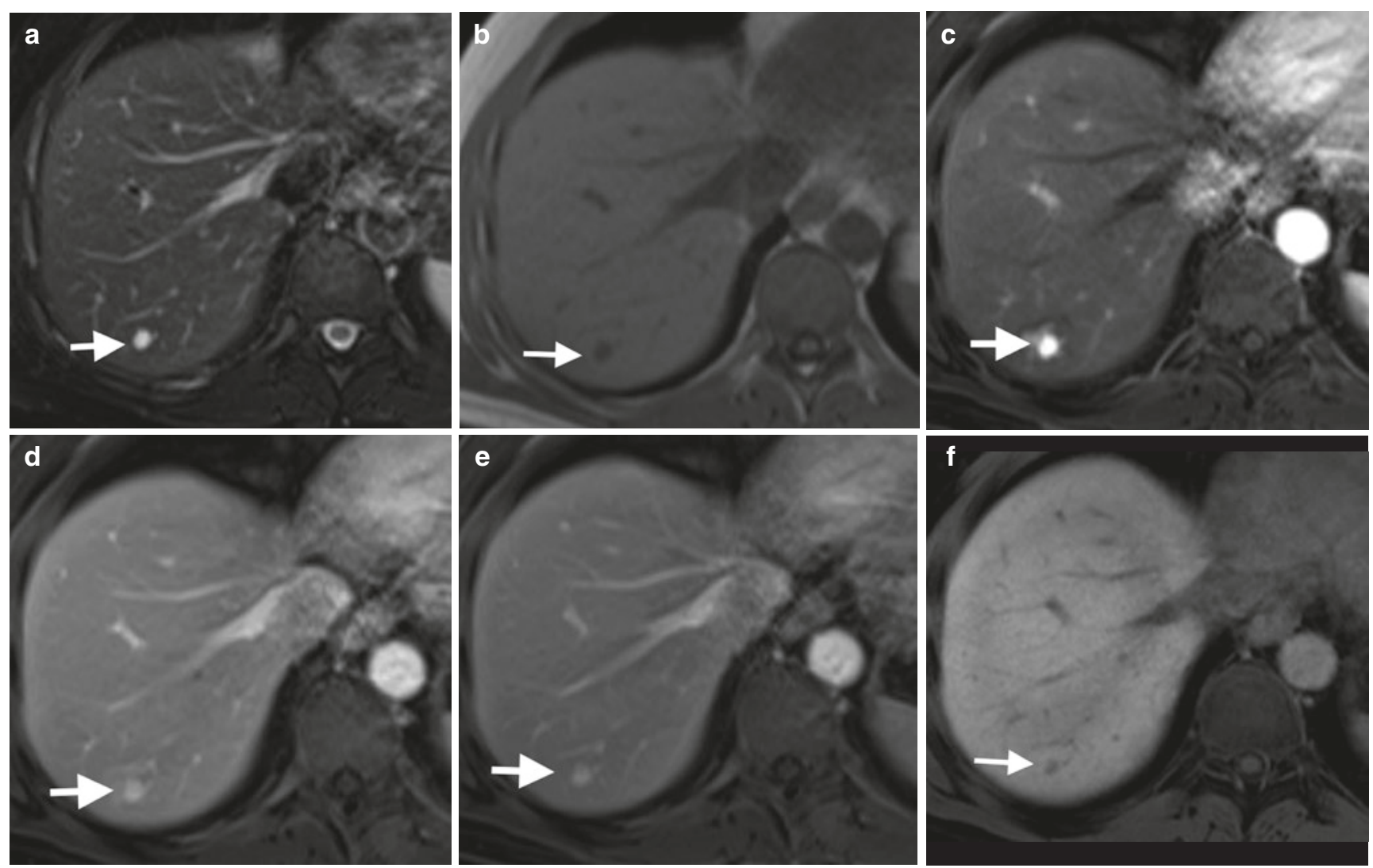

Fig. 17.3 Hemangioma type 1. Liver-specific MR contrast agent. A 45-year-old woman with incident lesion (arrows) in the right lobe of the liver. This appears as (a) high signal intensity on T2-weighted imaging and (b) low signal intensity on T1-weighted imaging and (c-e) shows

uniform enhancement on dynamic T1-weighted contrast-enhanced imaging, isointense to the vascular signal at all phases. The lesion appears (f) hypointense in the hepatobiliary phase of gadoxetic acidenhanced MRI
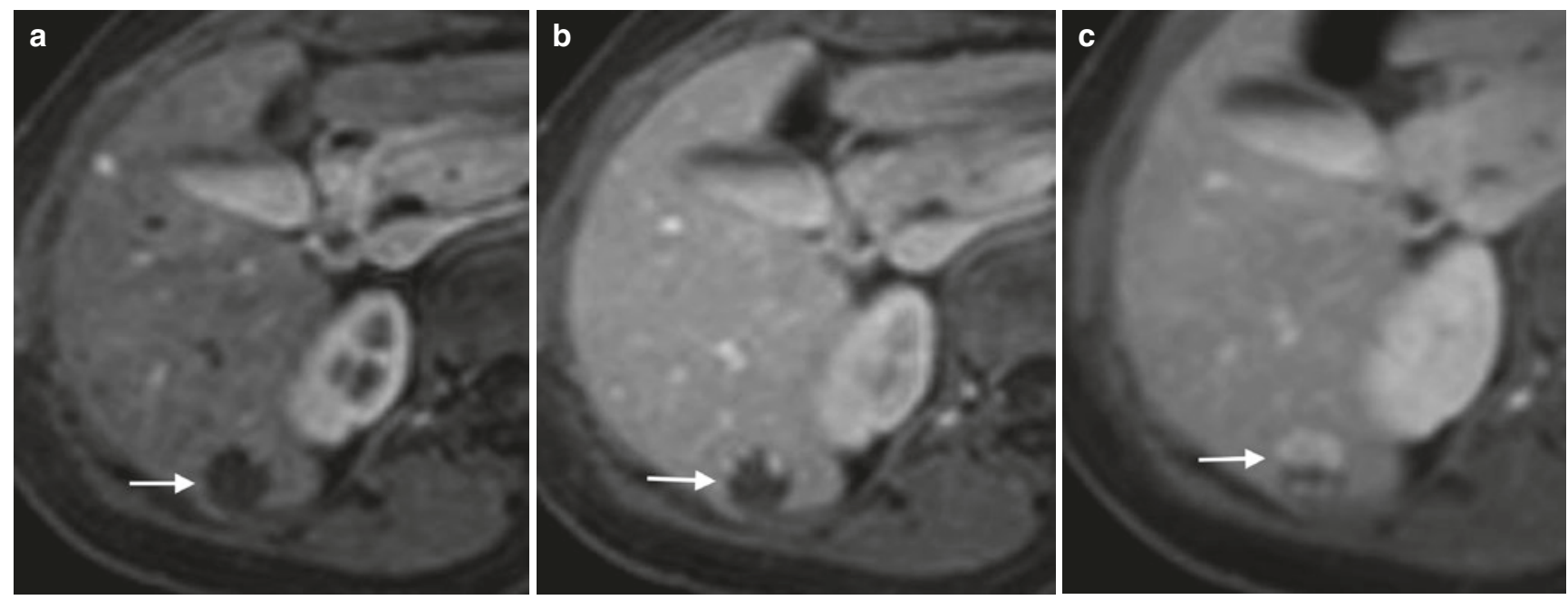

Fig. 17.4 Hemangioma type 3: nonspecific gadolinium chelate. (a-c) T1-weighted dynamic enhanced T1-weighted GRE in the (a) arterial enhancement of the lesion with centripetal filling. There is incomplete and (b) portal venous and (c) delayed phase shows nodular peripheral 

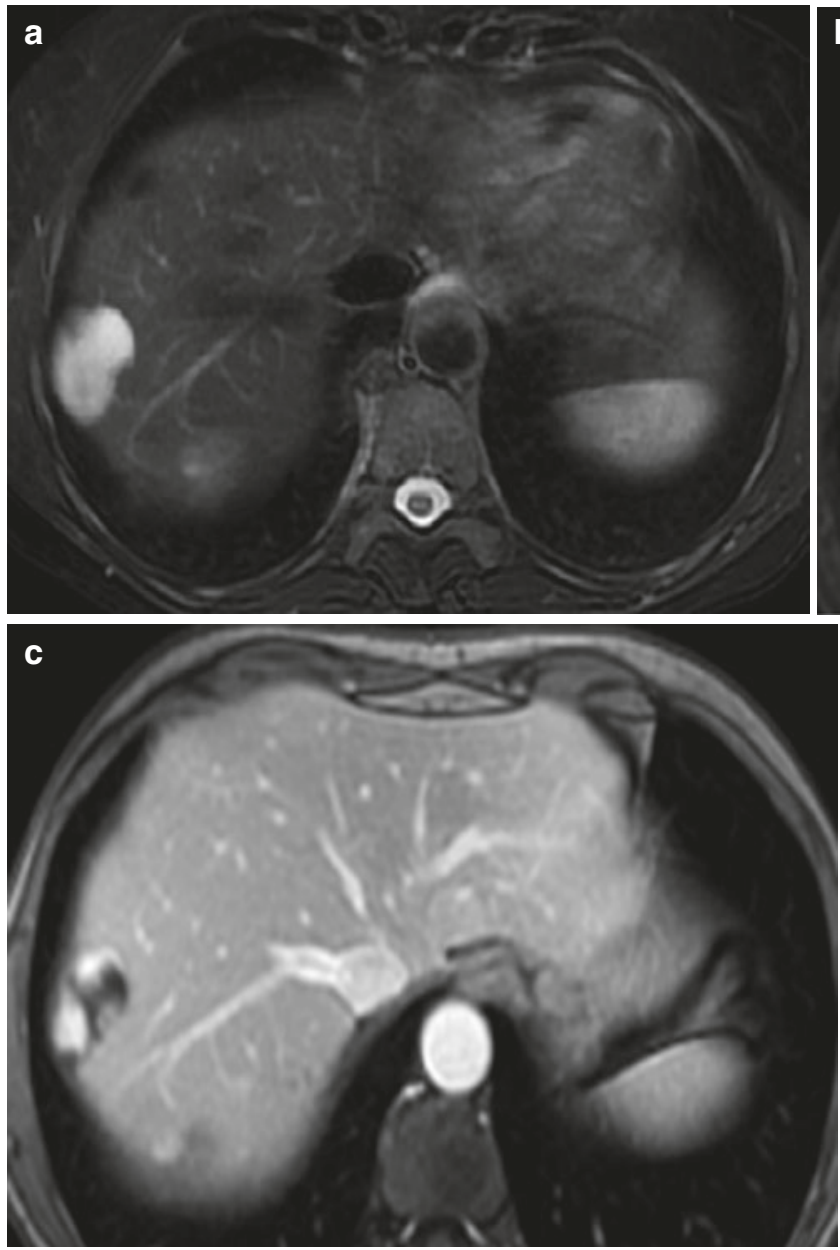

Fig. 17.5 Hemangioma type 3: liver-specific MR contrast agent. (a) T2-weighted TSE shows a large lobulated lesion of very high signal intensity. (b-d) Dynamic gadoxetic acid-enhanced imaging shows peripheral nodular enhancement in the arterial (b) and venous phases

images. Due to the prominent arterial vascular supply, FNH demonstrates marked homogenous enhancement during the arterial phase of contrast-enhanced CT/MR imaging, which becomes rapidly isodense/isointense to liver parenchyma in the portal venous phase [34]. The central scar often showed delayed enhancement (Fig. 17.6) [33] because of its vascular component. Another key feature is that other than the scar, FNH are usually homogeneous in appearance compared with the heterogeneous appearance encountered in fibrolamellar HCC.

Using liver-specific MR contrast agents, FNH frequently shows enhancement on delayed images after administration of hepatobiliary contrast agents (such as gadoxetic acid or gadobenate dimeglumine) because of the presence of normal biliary ductules within the lesion and the expression of OATP receptors (Fig. 17.6). However, the uptake of hepatobiliary contrast agents within FNH may be rarely heterogeneous or absent [36]. Nonetheless, a recent meta-analysis showed that the lesion $\mathrm{T} 1$ isointensity or hyperintensity at delayed hepatobiliary phase MRI
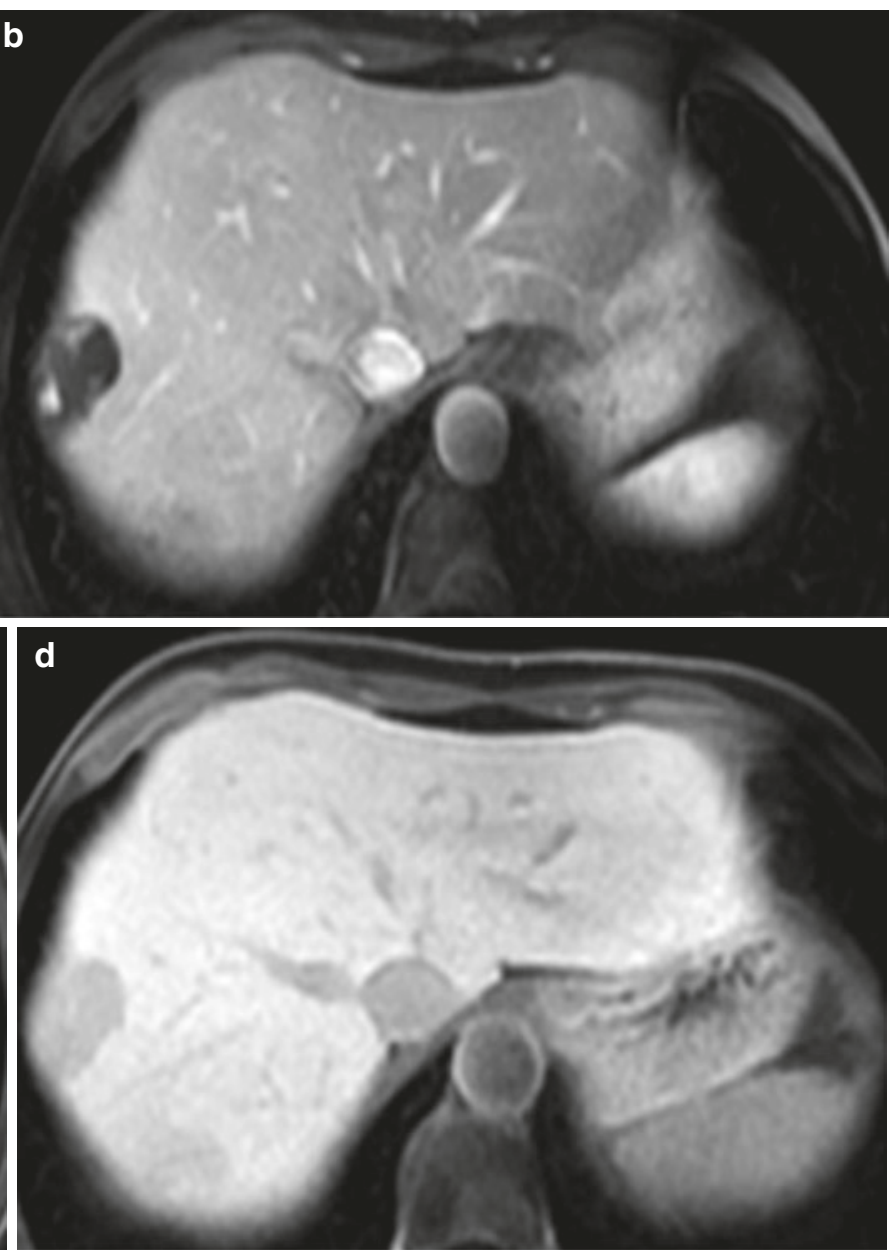

(c). In the hepatobiliary phase (d) there is marked hypointensity of the lesion due to lack of hepatocellular uptake in the lesion and enhancement of surrounding liver parenchyma

has a high sensitivity (91-100\%) and specificity (87$100 \%$ ) for diagnosing FNH [36]. This feature can be helpful for differentiating FNH from hypervascular metastases or hepatic adenomas (HCA) and hepatocellular carcinomas (HCC) (which do not usually take up liver-specific agents) $[31,37]$. However, it should be noted that some HCAs (particularly inflammatory HCA and beta-cateninactivated HCA) and HCC can appear isointense or hyperintense at delayed imaging after hepatobiliary contrast media administration. While differentiating FNH from variants of HCA remains challenging, it has been suggested that the presence of contrast washout (i.e., lesion hypointensity compared to liver parenchyma) of HCC in the portal venous or transitional phase of dynamic contrast enhancement can be used to distinguish between HCC (that shows contrast uptake in the hepatobiliary phase) and FHN nodules. The majority of FNH tend to remain static in size, although FNH may increase in size on follow-up (3-11\%), although oral contraceptives do not appear to stimulate FNH growth $[38,39]$. 

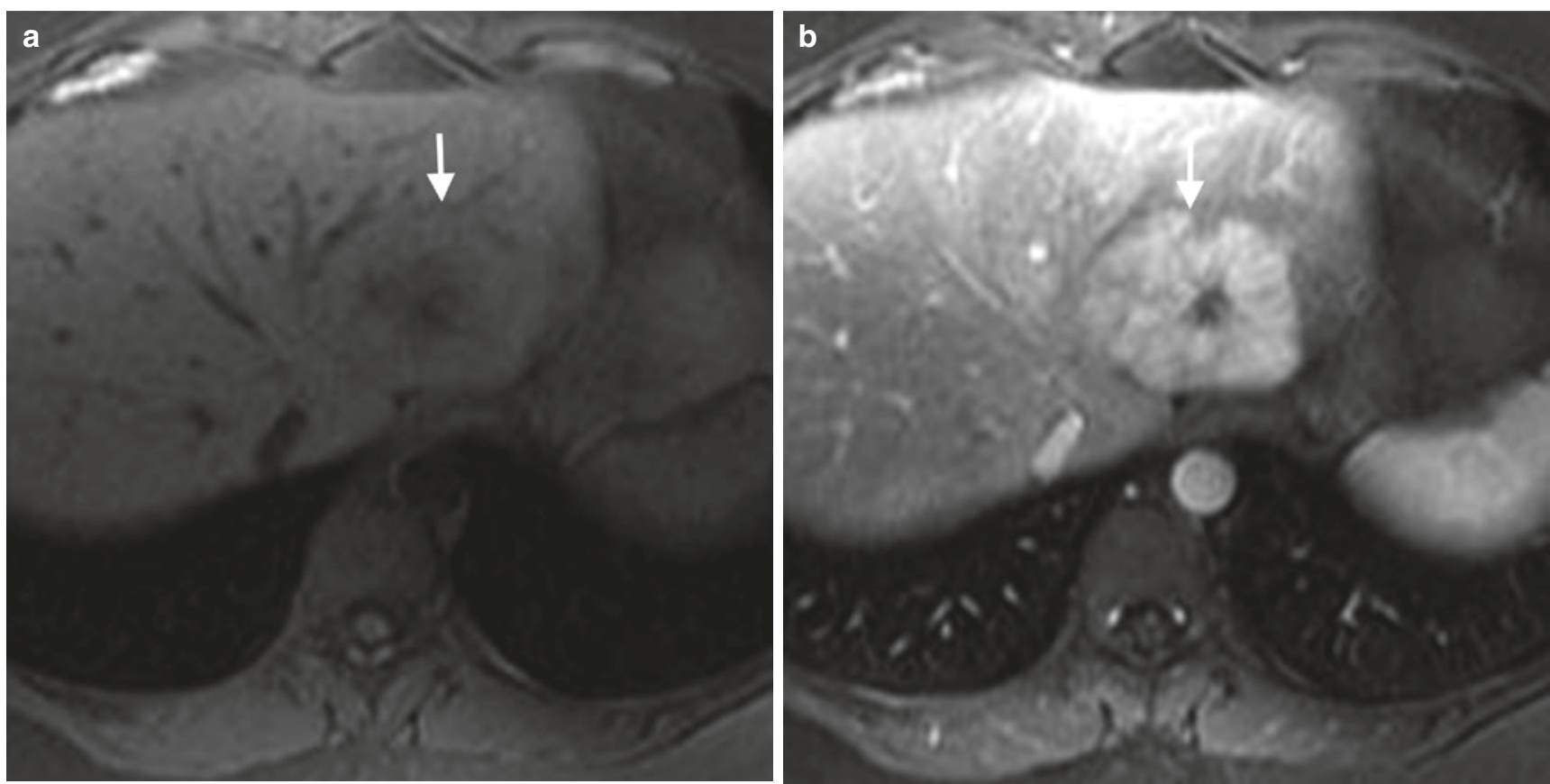

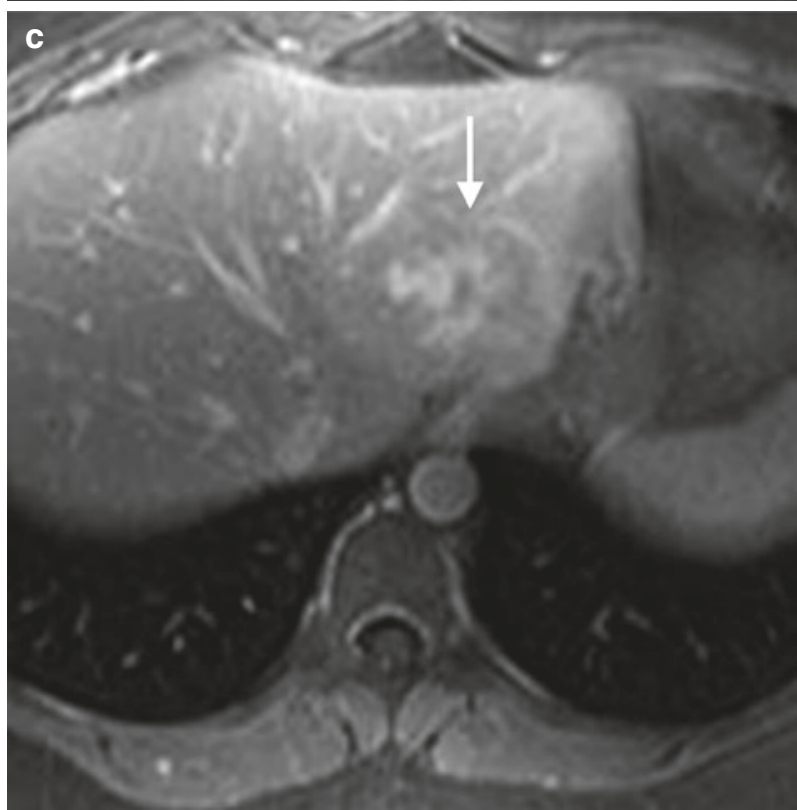

Fig. 17.6 FNH. Incidental lesion in the left lobe of the liver (arrows) (a) Pre-contrast T1-weighted image shows an isointense lesion with a central hypointense scar, which shows minimal mass effect upon adjacent vasculature. (b) Arterial phase T1-weighted contrast-enhanced image shows hypervascularity of the lesion. (c) T1-weighted delayed phase imaging after contrast shows that the lesion is now predominantly

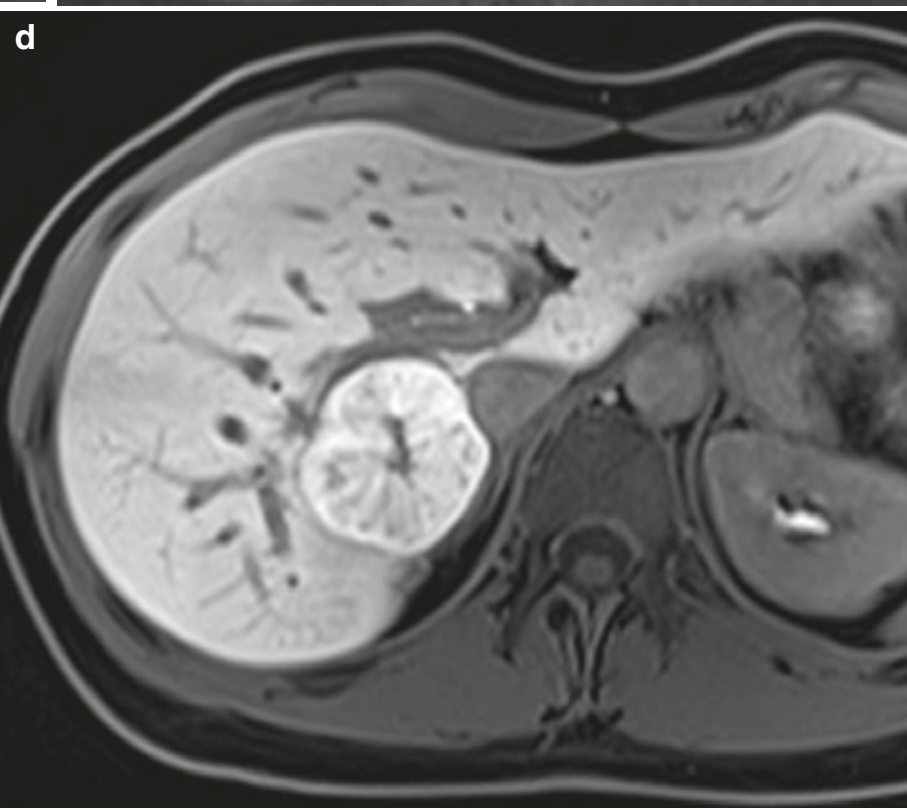

isointense to the liver but with late enhancement of the (vascular) central scar. The enhancement pattern is typical for FNH. (d) Hepatobiliary phase imaging of another FNH: homogenous uptake of the liver-specific MR contrast agent, the spoke-wheel central scar is typically not enhanced

The imaging features of HCA are heterogeneous and varied. HCA are often hypervascular and may appear heterogeneous due to the presence of fat, necrosis, or hemorrhage $[39,40]$. T1-weighted chemical shift or DIXON imaging is useful for detecting intratumoral fat, while the presence of high T1-signal before contrast administration will raise the suspicion of spontaneous hemorrhage. The reader should be
Hepatocellular adenoma (HCA) is uncommon, but has an association with oral contraceptive and anabolic steroid usage. Histologically, HCA is composed of cells resembling normal hepatocytes but lacking bile ducts, which distinguishes them from FNH [39]. 
familiar with the differential diagnoses of fat containing focal liver lesions on MRI, which include focal fat infiltration, HCA (particularly the HNF1A inactivating subtype), hepatocellular carcinoma (usually well differentiated), angiomyolipoma, lipoma, teratoma, and liver metastases from fat containing malignancies (e.g., liposarcomas). The presence of intratumoral fat helps to narrow the differential diagnosis of a hypervascular lesion, as hemangioma can be excluded and metastases and FNH rarely contain fat.

On dynamic contrast-enhanced CT or MR, adenomas usually show marked arterial-phase enhancement, with rapid transition to either iso- or hypoattenuating/intense to hepatic parenchyma on portal venous phase imaging. Our understanding of the molecular aberrations associated with HCA has improved our understanding of HCA subtypes, which is linked to risk factors, histological features, clinical presentation, and imaging appearances $[41,42]$. The latest molecular classification categorizes HCA into the following six subgroups: HNF1A-inactivated HCA, inflammatory HCA, CTNNB1-mutated HCA in exon 3, CTNNB1 mutated in exon 7 and $8 \mathrm{HCA}$, sonic hedgehog HCA, and unclassified HCA [43, 44].

What is important for radiologists? Inactivating mutations of hepatocyte nuclear factor 1 alpha (HNF1A) are observed in $40-50 \%$ of HCA. HNF1A-inactivated HCA usually contains fat as evidenced by diffuse and homogenous signal loss on chemical shift T1-weighted imaging (Fig. 17.7). They return variable T2 signal. At contrast-enhanced T1-weighted MRI, they are hypervascular, often with contrast washout in the portal venous or delayed phase. They are typically hypointense on hepatobiliary-phase MRI using liver-specific contrast medium. HNF1A-inactivated HCAs have a very low risk of malignant transformation.

Inflammatory HCA accounts for $35-45 \%$ of HCA cases. Obesity and a history of oral contraceptives intake are risk factors for their development. Inflammatory HCA appear strongly hyperintense on T2-weighted MRI, which may be diffuse or rim-like in the periphery of the lesion (Atoll sign). Intralesional fat is uncommon and, when present, is often patchy or heterogeneous. On contrast-enhanced imaging, there is usually intense arterial enhancement, with persistent enhancement on delayed phase imaging (Figs. 17.8 and 17.9). Although the majority of inflammatory HCA are hypointense on hepatobiliary phase using liver-specific contrast media, about $30 \%$ may appear iso- or hyperintense. Inflammatory HCA may also harbor activating mutations of b-catenin in exon 3 and are therefore at risk of malignant transformation.

Mutations of catenin b1 (CTNNB1) in exon 3 (coding for b-catenin) are seen in 10-15\% of HCA. These are associated with a higher risk of malignant transformation. By contrast, a subset of HCA (5-10\%) is associated with mutations of CTNNB1 in two hot spots in exon 7 and 8, which does not confer an increased risk of malignancy. These variants of HCA do not have typical imaging features and may be difficult to differentiate from HCC or FNH. HCA with mutations of catenin b1 may also show contrast uptake in the hepatobiliary phase of MRI using liver-specific contrast media.

Activation of sonic hedgehog pathway occurs in approximately $5 \%$ of HCA. As these are relatively uncommon, the spectrum of imaging features associated with these is yet to be fully described. Nonetheless, these lesions have a higher propensity to undergo spontaneous hemorrhage. About 7\% of HCA remains unclassified. These do not have typical clinical or imaging appearances.

Overall, the imaging features at MRI, including their appearances using liver-specific MR contrast agents (gadobenate, gadoxetic acid) are helpful in distinguishing between FNH and HCA. In the hepatobiliary phase of contrast enhancement, FNH typically show contrast uptake, whereas NHF1A-inactivated HCA and the majority of other HCA subtypes do not [44]. Of note is that diffusion-weighted MRI has little value in helping to distinguish between HCA and FNH or HCC because of the substantial overlap in the ADC values.

\subsubsection{Biliary Hamartomas (Von Meyenburg Complex)}

Bile duct hamartomas are congenital malformations of the ductal plate without connections to the bile ducts. They are usually discovered incidentally at abdominal imaging. Although of no clinical significance, they can mimic disseminated small liver metastases in the patient with cancer. Biliary hamartomas are typically small (5-10 $\mathrm{mm}$ in size) and usually widely distributed in both lobes of the liver. On ultrasound, they appear as small hyperechoic or hypoechoic lesions and can demonstrate ringing artifacts (comet tail appearance). On CT, they appear as small cystic lesions of round, oval, or irregular shape without contrast enhancement, although thin rim enhancement may sometimes be present, thus mimicking hypovascular liver metastases [40]. When enhancement is present, it is usually very thin $(\leq 2 \mathrm{~mm})$ and observed only on equilibrium-phase images, related to the fibrous component of the lesions [45]. On MRI, biliary hamartomas appear low signal intensity on T1-weighted imaging and high signal intensity on T2-weighted imaging (Fig. 17.10). They are best observed on maximum intensity projections MRCP sequences as high signal intensity foci without connection to or associated abnormalities of the intrahepatic ducts. Occasionally, bile duct hamartomas can 

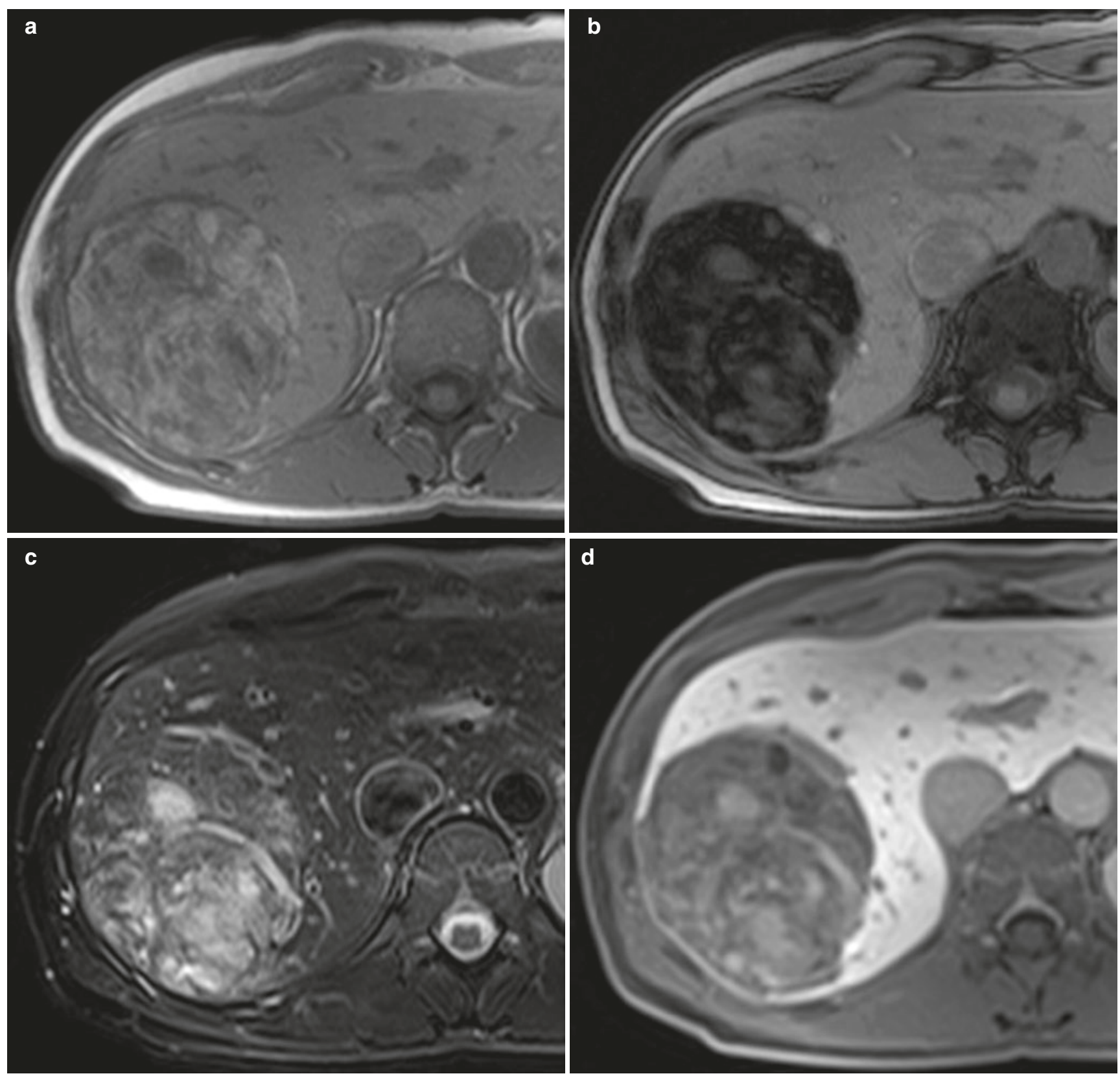

Fig.17.7 Adenoma (HNF1A subtype). (a) T1-weighted in-phase GRE image demonstrates a very large mass in a young woman. The mass is inhomogeneous and shows bright spots. (b) There is typical signal intensity drop on the opposed-phase image indicative of intratumoral

be very large, up to $20 \mathrm{~cm}$, and be symptomatic from internal hemorrhage or pressure on adjacent structures [46]. Differential diagnoses of biliary hamartomas include peribiliary cysts (predominantly perihilar distribution in patients with liver parenchymal disease), polycystic disease, and Caroli's disease (cysts communicate with bile ducts and are associated with bile duct abnormalities). They can also mimic liver abscesses in the appropriate clinical setting.

fat. (c) The T2-weighted TSE shows moderate hyperintensity. (d) On the gadoxetic acid-enhanced images in the hepatobiliary phase, there is little to no enhancement

\subsubsection{Hepatic Abscess and Echinococcus}

The appearances of hepatic abscesses on imaging depend on etiology (peribiliary abscesses tend to be small and scattered adjacent to the biliary tree; hematogenous distribution via the hepatic artery or via the portal vein in appendicitis or diverticulitis tends to lead to larger lesions diffusely spread in the liver). US reveals a cystic lesion with internal 

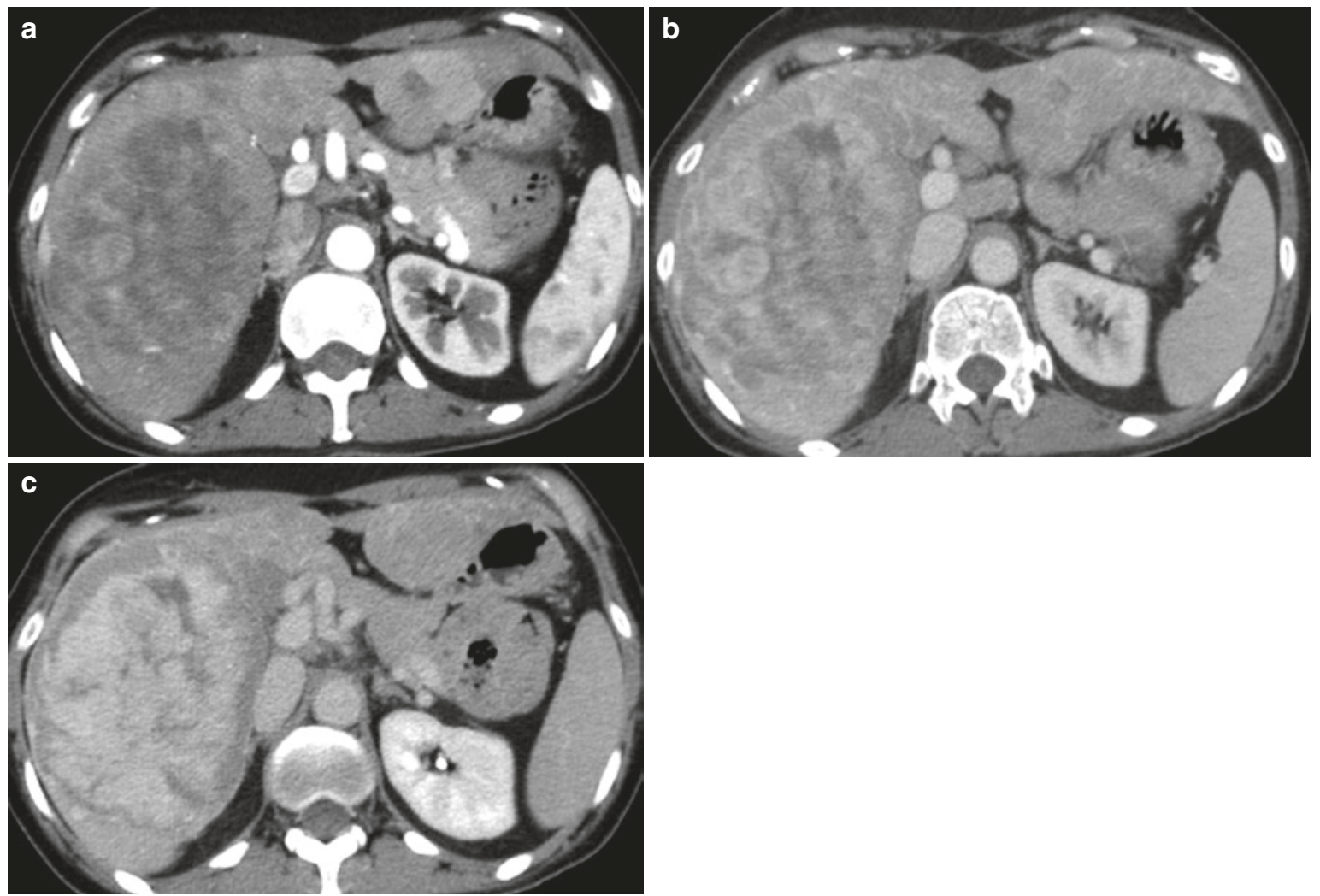

Fig. 17.8 Adenoma: inflammatory type. (a-c) Arterial (a) venous (b) phase CT shows strong and progressive contrast enhancement of the lesion, which retains enhancement in the delayed phase (c), which is typical for peliotic changes in inflammatory adenoma
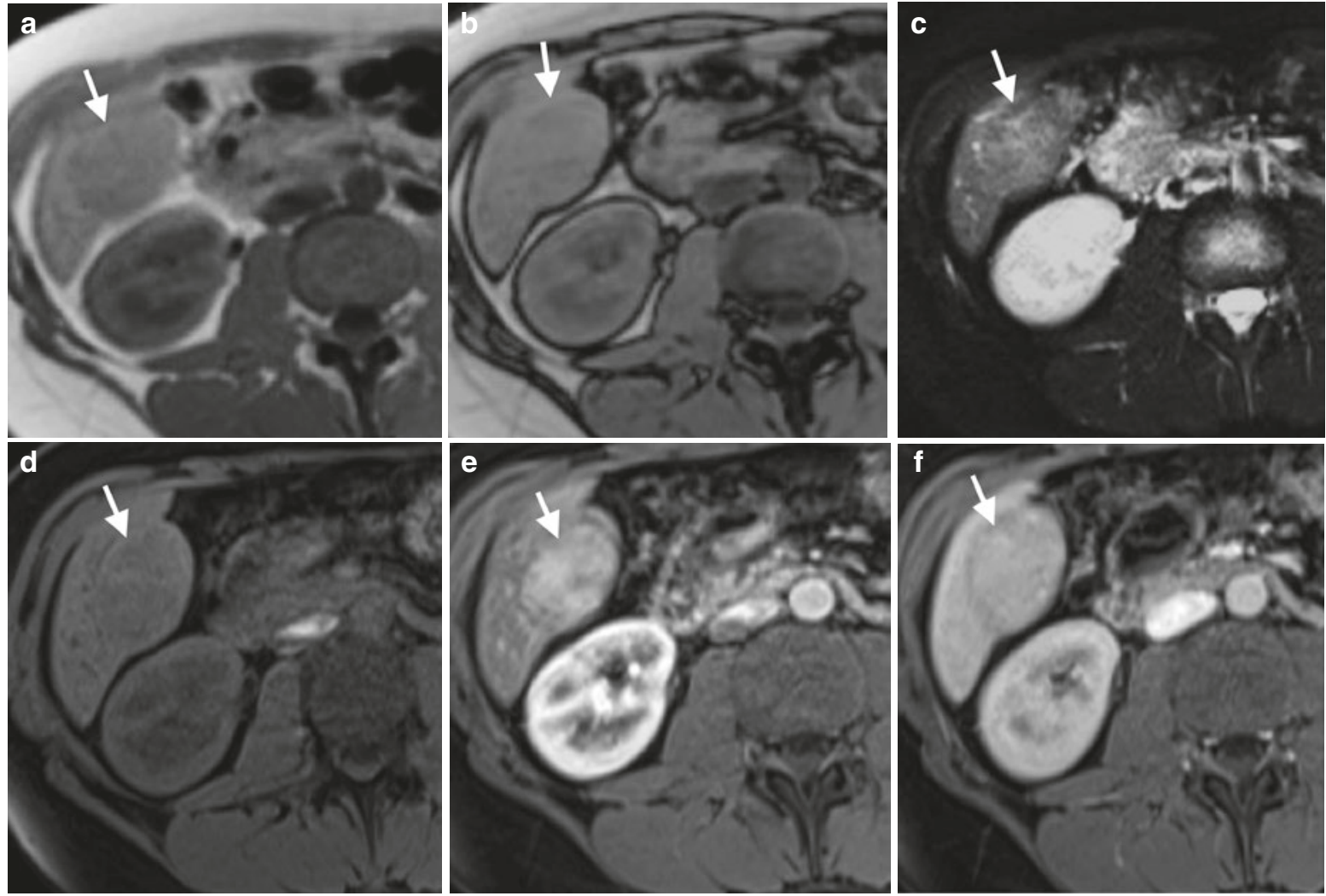

Fig. 17.9 Adenoma (inflammatory type) in a young female presenting with vague upper quadrant pain. (a) In- and (b) opposed-phase T1-weighted imaging shows no significant intralesional fat. The nodule is (c) mildly hyperintense on T2-weighted imaging. (d-f) Pre-contrast, post-contrast arterial phase, and delayed phase images show avid arterial enhancement, which persists. Surgical resection confirmed an inflammatory adenoma 


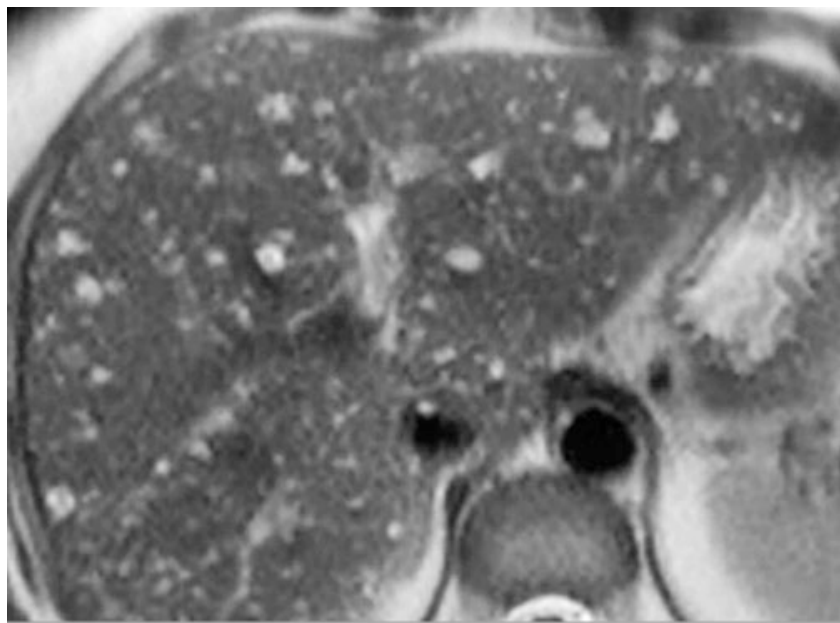

Fig. 17.10 Biliary hamartomas (von Meyenburg complex). A middleaged woman was referred to MRI following an ultrasound examination. There are multiple foci of high T2-weighted signal within the liver, suggestive of biliary hamartomas

echoes. On CT, hepatic abscesses are hypodense lesions with capsules that may show enhancement (Fig. 17.11); cluster sign may be noted when multiple abscesses are present [47]. CT appearance of hepatic abscess is nonspecific and can be mimicked by cystic or necrotic metastases. Hence, appropriate clinical and laboratory corroboration is vital toward making the right radiological diagnosis. Though present in only a small minority of cases, central gas is highly specific for abscess. On MR imaging, hepatic abscesses are hypointense relative to liver parenchyma on T1-weighted images and markedly hyperintense on T2-weighted images, often surrounded by a local area of slight T2 hyperintensity representing perilesional edema, which may also show increased enhancement after contrast administration.

Amebic liver abscess is nonspecific. It usually appears as a solitary, hypodense lesion, with an enhancing wall that may be smooth or nodular, and is often associated with an incomplete rim of edema. With MR imaging, lesions are hypointense on T1-weighted images and heterogeneously hyperintense on T2-weighted images [48].

On CT scan, involvement of liver by Echinococcus granulosus (hydatid cyst) can manifest as unilocular or multilocular cysts with thin or thick walls and calcifications, usually with daughter cysts seen as smaller cysts, with septations at the margin of or inside the mother cyst (i.e., this appearance is quite different from a "usual" multicystic tumor). On MR imaging, the presence of a hypointense rim on $\mathrm{T} 1$ - and T2-weighted images and a multiloculated appearance are diagnostic features.

\subsection{Malignant Primary Tumors}

\subsubsection{Hepatocellular Carcinoma}

HCC is the most common primary liver cancer, with the highest incidence in Asia and the Mediterranean. In European countries, HCC is found mostly in patients with chronic liver disease (particularly hepatitis B or C, liver cirrhosis, or hemochromatosis). At histopathology, HCC is characterized by abnormal hepatocytes arranged in trabecular and sinusoidal patterns. Lesions may be solitary, multifocal, or diffusely infiltrating.

There is wide varying appearances of HCC on imaging. An early $\mathrm{HCC}$ occurring within at risk population is typically small $(<3 \mathrm{~cm})$ and has a homogenous appearance. By contrast, late presentation disease (including tumor in noncirrhotic patients) is characterized by more advanced disease, presenting as a larger heterogeneous lesion. US is frequently used for disease screening and surveillance of cirrhosis patients. The appearance of HCC on US is variable, with iso-, hypo-, or hyperechogenicity (increased echogenicity is often due to intratumoral fat). Smaller lesions are typically homogeneous and larger lesions heterogeneous. A surrounding fibrous capsule is often present and characteristic for HCC, appearing as a hypoechoic rim surrounding the lesion.

On unenhanced CT images, most HCCs are hypo- or isodense (the latter particularly if small). The presence of intratumoral fat can lower CT attenuation and is suggestive of primary hepatocellular tumors in the appropriate clinical settings. Due to their altered and predominant arterial supply, HCCs enhance avidly in the arterial phase of contrast enhancement, becoming iso- or hypodense with the liver parenchyma in the portal venous phase of enhancement. Delayed phase images show most HCC lesions as hypodense compared with surrounding liver. The washout of contrast in these tumors is a diagnostic characteristic of HCC (Fig. 17.12). Small HCCs may have a nodule-in-nodule appearance on CT and MR images, especially when the disease develops within a regenerative or dysplastic nodule (Fig. 17.13). At MR imaging, such a nodule can exhibit higher signal intensity on T2-weighted images and display hypervascularity on arterial-phase images.

Multiphase imaging after contrast administration on CT helps to optimize the detection and characterization of HCC. Late arterial-phase imaging is the most sensitive for detecting small lesions [6, 49, 50]. A venous phase is always necessary for tumor detection/characterization and 


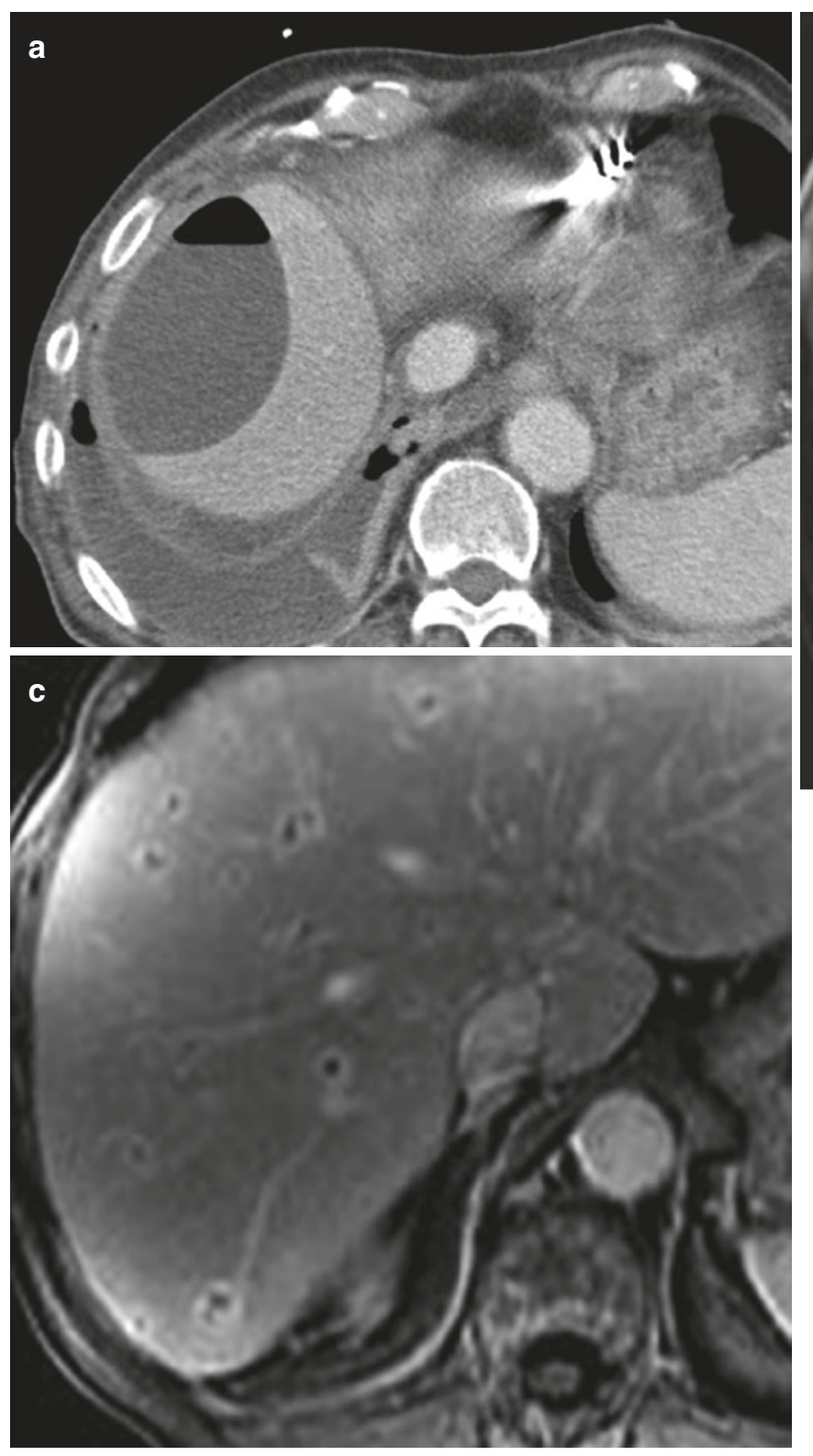

Fig. 17.11 Abscesses. (a) Typical large subcapsular abscess with an air-fluid level and a reactive pleural effusion. (b, c) Another patient with fever and right upper quadrant pain. T1-weighted contrast-enhanced images in the (b) arterial and (c) portal venous phase demonstrate mul-

assessment of venous structures (Fig. 17.12), as well as other abdominal organs. The delayed phase imaging (e.g., at 2-3 min) can occasionally help to detect a lesion that may be missed [51]. Much more important is that it can help to make a firm diagnosis of HCC by showing typical lesion contrast washout, if it had not been present in the portal venous phase [52]. Unenhanced images are important for identifying hyperdense siderotic nodules and for detecting hypodense intratumoral fat. Unenhanced images are also useful for tumor follow-up after chemoemboliza-

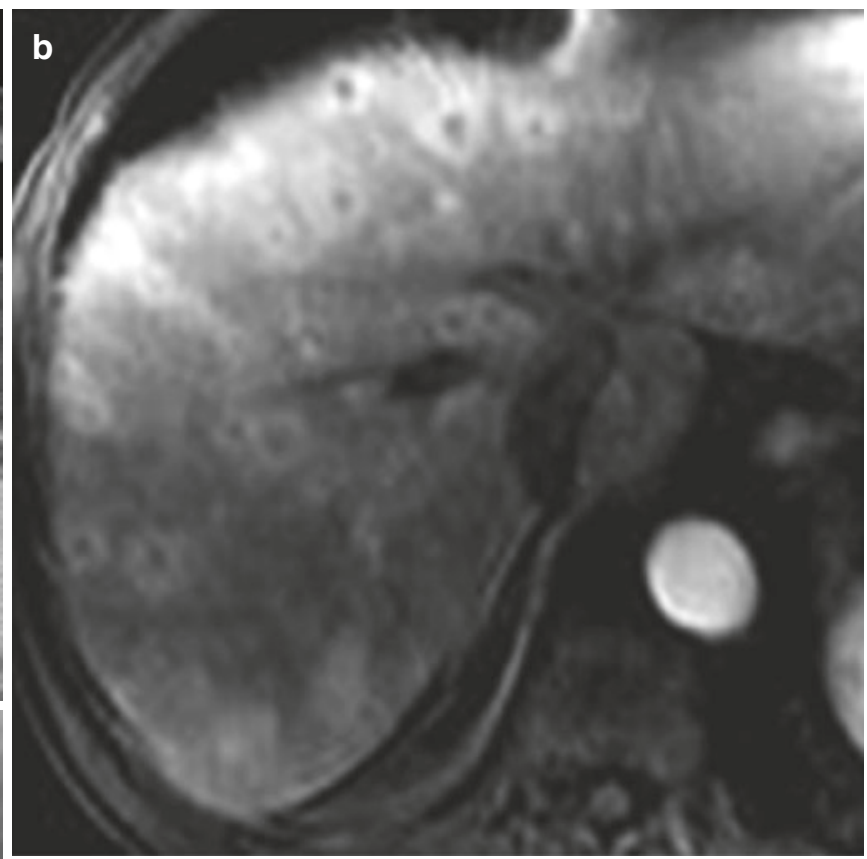

tiple ring-enhancing lesions in both lobes of the liver. In the arterial phase, there is also associated increased parenchyma enhancement surrounding many of the lesions. The appearance is consistent with multiple hepatic abscesses

tion or after tumor ablation. For these reasons, a three- to four-phasic MDCT protocol is utilized at most centers to evaluate $\mathrm{HCC}$.

The reliance on focal hypervascularity in the arterial phase can lead to false-positive diagnosis of HCC [53]. Transient focal enhancement of liver parenchyma during arterial phase, also termed transient hepatic attenuation differences (THAD), can lead to a false diagnosis of HCC. In cirrhotic patients, transient focal enhancement is most often caused by arterial-portal shunting, resulting in inappropri- 

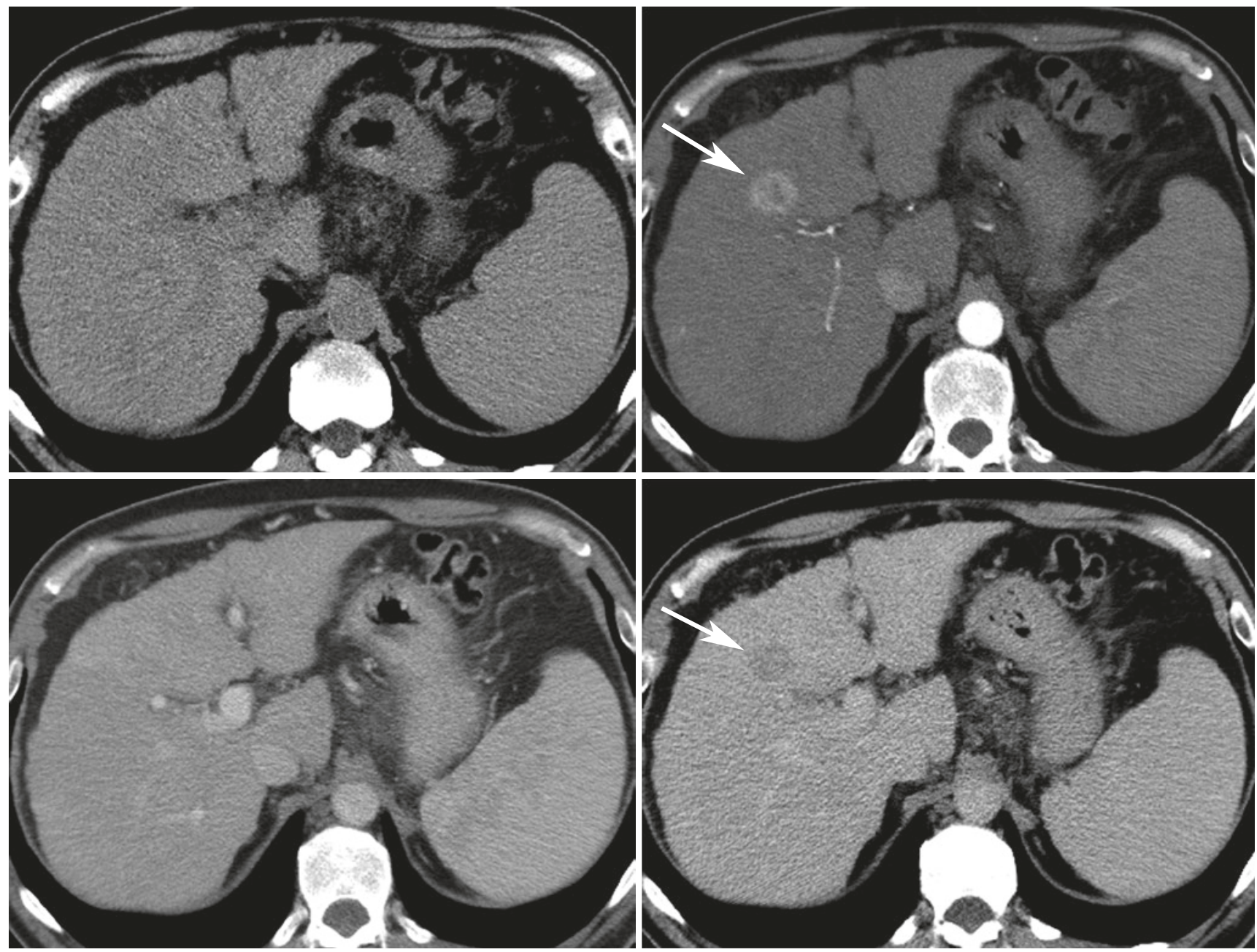

Fig. 17.12 HCC: quadruple-phasic CT for detection and characterization. (a) Non-contrast CT shows liver cirrhosis and splenomegaly. In segment 4, a lesion is only faintly seen. (b) In the late arterial phase, a hypervascular HCC is depicted in segment 4 (arrow). (c) In the venous

phase, the lesion is not visible. (d) The delayed phase scan reveals washout of the lesion, which is now hypoattenuating (arrow). The combination of arterial hypervascularity and washout is a very specific sign of malignancy

ately early focal areas of portal venous distribution enhancement in the liver. THAD are usually peripherally located in the liver, appear wedge shaped, and may be poorly circumscribed. Subcapsular lesions that do not exhibit mass effect or a round nature should be carefully evaluated before suggesting the diagnosis of HCC. THAD are not associated with lesion hypodensity in the portal venous or delayed phases of contrast enhancement.

The combination of hyperdensity on arterial-phase images combined with washout to hypodensity on venous- or delayed phase images, although not sensitive (33\%), is highly specific (100\%) for the diagnosis of HCC [54] (Fig. 17.11). However, a small proportion of HCC can be isovascular or hypovascular compared with the liver, which can be difficult to diagnose. The typical MR imaging features of larger HCC include a fibrous capsule/ pseudocapsule, intratumoral septa, daughter nodules, and tumor thrombus (Fig. 17.14) [55]. These lesions are often heterogeneous in appearances (mosaic architecture) on both CT and MR [56]. Whereas most large HCC are hyperintense on T2-weighted images, smaller lesions, measuring even 3-4 cm, can appear isointense or hypointense. On T1-weighted images, HCC shows variable signal intensity relative to hepatic parenchyma. A tumor capsule/pseudocapsule may be seen on T1-weighted and, less commonly, as hypointense on T2-weighted imaging.

Conventional gadolinium contrast imaging in HCC parallels the features described for CT, with characteristic early peak contrast enhancement and delayed phase tumor contrast washout of the nodular solid components, as well as late T1 enhancement of the capsule/pseudocapsule. Liverspecific MR contrast agents (gadoxetic acid or gadobenate 

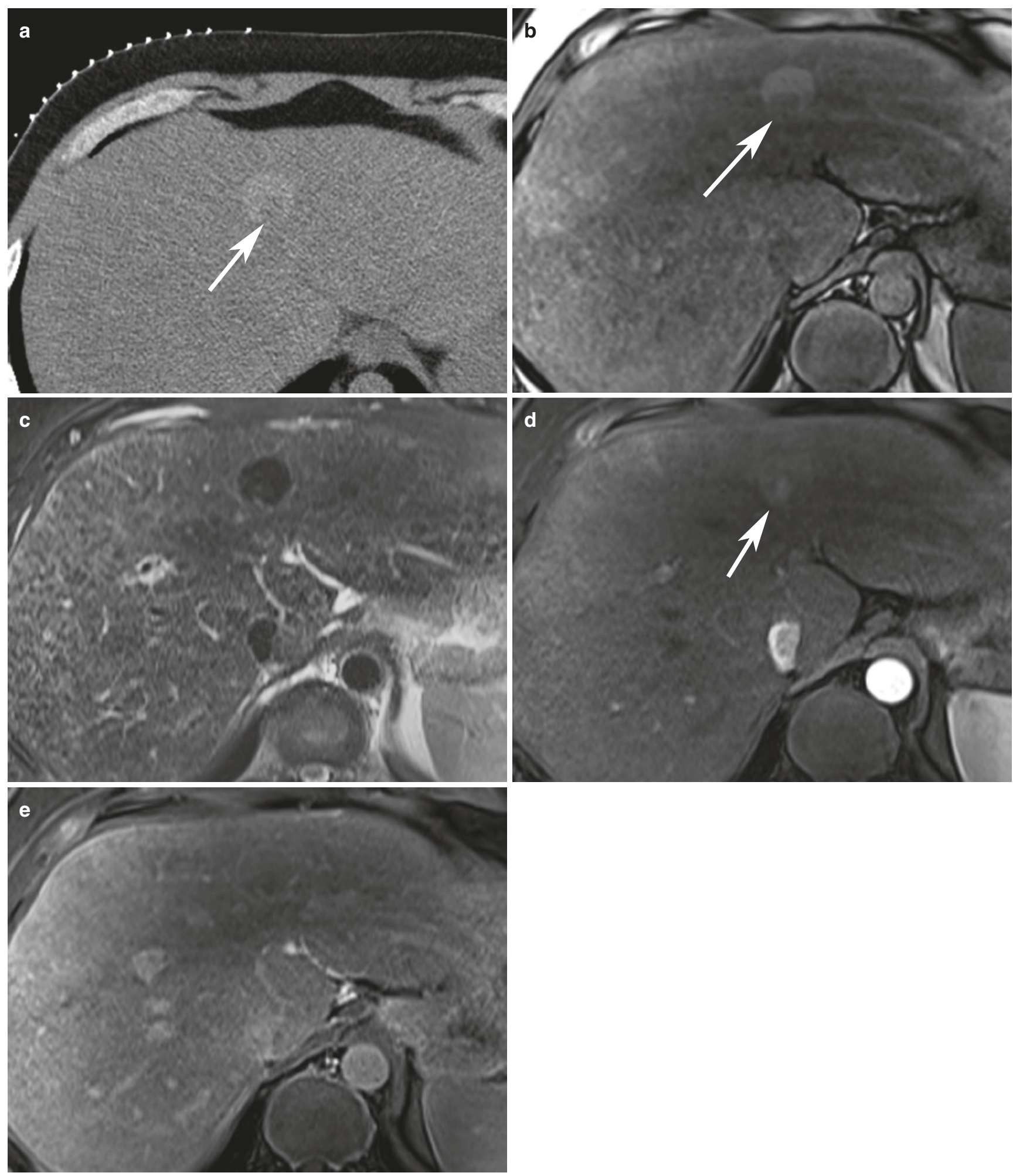

Fig. 17.13 HCC with nodule-in-nodule appearance. (a) Unenhanced CT shows a siderotic (hyperattenuating) large nodule, which contains a low-density (non-siderotic) focus (arrow). (b) On T1-weighted GRE opposed-phase image, the marginal nodule shows low signal intensity (arrow). (c) The large nodule shows siderosis on T2-weighted TSE

images, but the marginal focus displays higher SI. (d, e) Dynamic gadolinium-enhanced T1-weighted GRE images show (d) arterial hypervascularity of the malignant focus (arrow) and (e) washout in the equilibrium phase 

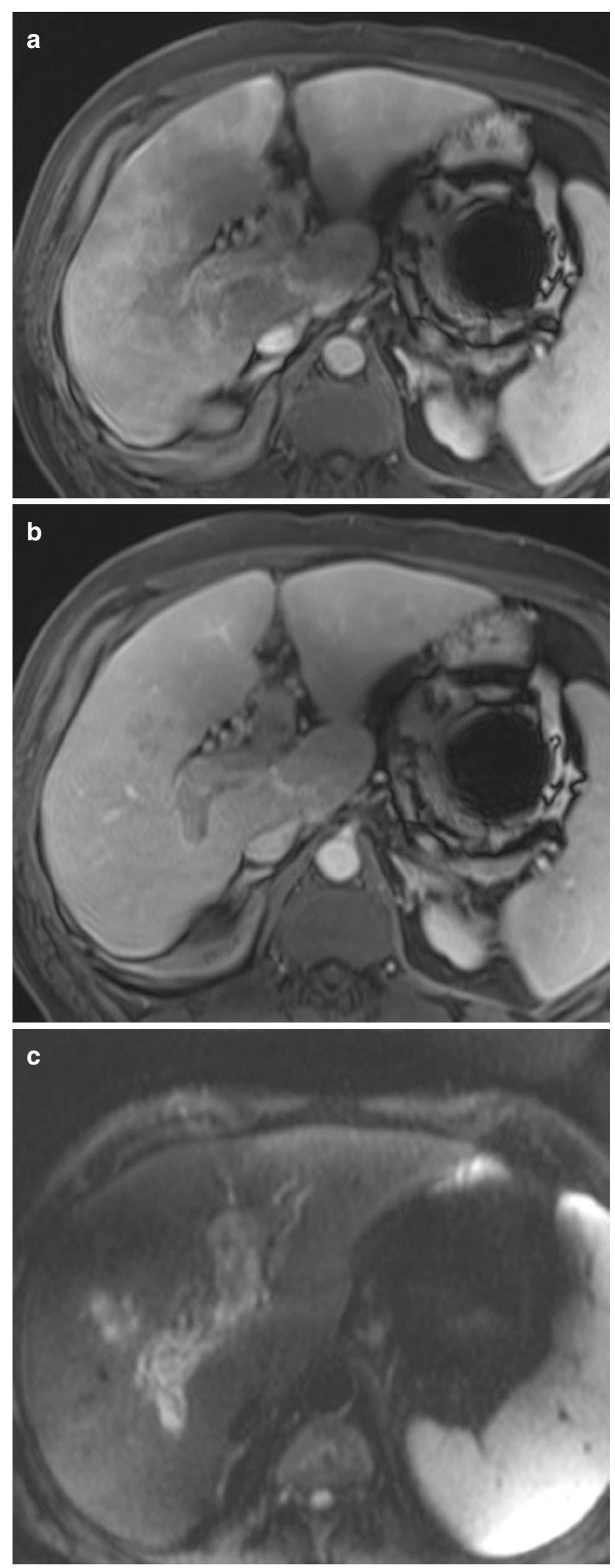

Fig. 17.14 Diffuse HCC in the right lobe with tumor thrombus in the portal vein. (a) Arterial phase and (b) venous phase T1-weighted GRE shows inhomogeneous enhancement and expansion of the portal vein. There is inhomogeneous enhancement of the right lobe, but no definite tumor is seen. (c) DWI shows a solid mass in the entire intrahepatic portal vein and part of the tumor in the right lobe dimeglumine) can be administered to provide arterial, portal venous, and equilibrium-phase imaging but has the added advantage of revealing additional characteristics at the delayed hepatobiliary phase of contrast enhancement. HCC typically do not show contrast retention of liver-specific contrast medium in the hepatobiliary phase, which can add confidence toward the detection and characterization of HCC (Fig. 17.15) [57]. It has been shown that using gadoxetic acid-enhanced MRI can improve the detection of small or early HCCs, as it is superior for detecting HCC measuring $<1-2 \mathrm{~cm}$ in size compared with CT [58]. In addition, subcentimeter lesions detected by gadoxetic acid-enhanced MRI are likely to be or can transform to become HCC within a short interval [59]. Hence, several evolving guidelines for the imaging evaluation of HCC are incorporating the role of liver-specific contrast media for the diagnosis of subcentimeter HCC. Subcentimeter HCC may be treated by locoregional therapy, thus avoiding the morbidity and mortality associated with radical surgery.

However, it is important to note some potential pitfalls of using liver-specific contrast media for HCC evaluation. Some benign regenerating nodules may appear hypointense at the hepatobiliary phase of contrast enhancement, although the majority appears isointense of the liver [60]. In addition, some well-differentiated or moderately differentiated HCC may appear isointense or hyperintense on delayed images due to higher levels of OATP1B3 and MRP3 receptor expression. For this reason, the use of ancillary imaging features at MRI can improve the confidence of HCC diagnosis. These include mild to high T2 signal intensity and impeded diffusion on high b-value DWI. The use of liver-specific contrast agents may also help toward the identification of isoenhancing or hypoenhancing HCC that do not show typical hypervascularity in the arterial phase of contrast enhancement. With regard to the use of diffusionweighted MRI for HCC evaluation, higher b-value (e.g., $800 \mathrm{~s} / \mathrm{mm}^{2}$ ) DWI may help in the identification of disease, particularly if the suspected nodule also demonstrates typical vascularity pattern at contrast-enhanced MRI. Because of background liver cirrhosis, higher-grade/poorly differentiated HCC are more likely to show impeded diffusion and lower ADC values compared with low-grade/well-differentiated HCC.

To summarize, many MR characteristics are often associated with HCC (arterial-phase hyperintensity, T2 hyperintensity, venous- or equilibrium-phase washout, lack of hepatobiliary MR contrast agent uptake on hepatobiliary phase images, and restricted diffusion on high-b-value DWI). However, for each of these findings, there is only $~ 60-80 \%$ sensitivity, and benign lesions show these findings in 16-65\% of cases, depending on finding, contrast agent used, and series reported $[60,61]$. Based on data from numerous studies, the American Association for the Study of Liver Disease (AASLD) and the European Association for the Study of the Liver (EASL) formed recommendations for the noninvasive 

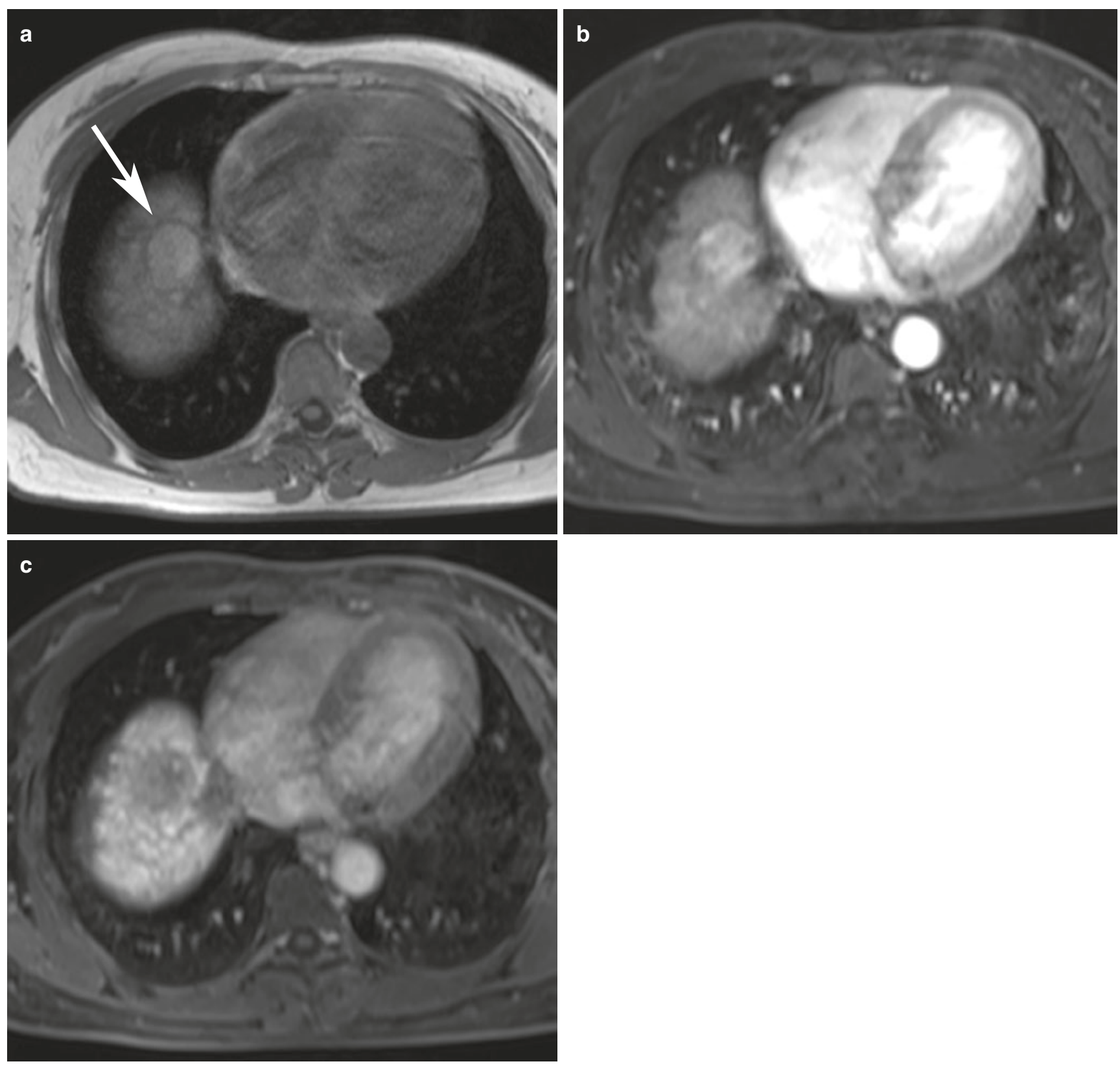

Fig. 17.15 HCC: MRI with liver-specific contrast agent (gadoxetic acid). (a) Axial T1-weighted GRE shows an encapsulated slightly hyperintense mass in the dome of the liver. (b) Gadoxetic acid-enhanced

diagnosis of HCC in patients with chronic liver disease [62]. Lesions more than $1 \mathrm{~cm}$ that demonstrate arterial-phase hypervascularity and venous- or delayed phase washout are triaged for treatment with a diagnosis of HCC. If only one of the two findings are present, then the guidelines require obtaining a different modality with contrast imaging to determine whether these findings can be verified. If the lesion remains atypical, then biopsy is recommended. If a suspected lesion is less than $1 \mathrm{~cm}$, the AASLD and EASL guidelines recommend repeating the examination at 3-month image shows strong enhancement in the arterial phase. (c) In the hepatobiliary phase after $20 \mathrm{~min}$, the lesion shows hypointensity due to lack of hepatocellular uptake

intervals, using the same imaging technology used to detect the lesion, to determine whether there is growth or changing in character. In following up patients with chronic liver disease, development of a new nodule with any of the MR signal abnormalities discussed above should be considered worrisome for HCC, even if they do not meet the AASLD [63] criteria for noninvasive diagnosis. These criteria were developed to be specific but are only approximately $70 \%$ sensitive [60]. It is essential for radiologists to also document the number and size of all lesions meeting criteria for HCC, 


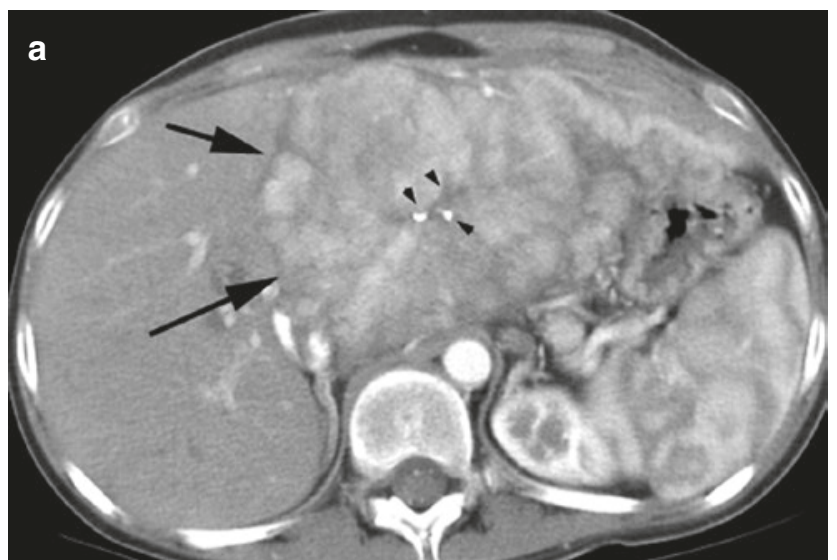

Fig. 17.16 Fibrolamellar HCC. (a) Arterial phase MDCT shows heterogeneously enhancing mass in the left lobe (arrows) with low attenuation central fibrous scar with calcifications (arrowheads). (b)

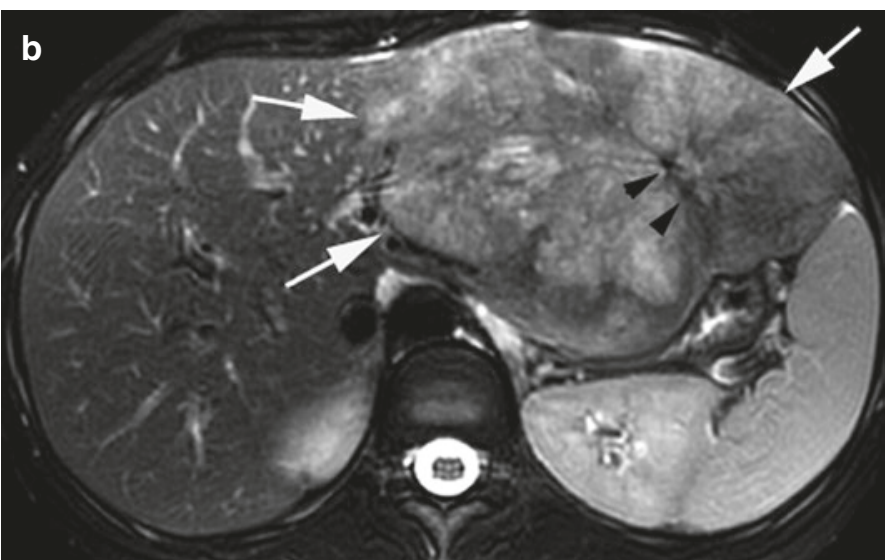

T2-weighted MRI shows large left lobe mass (arrows) with heterogeneous appearance and mild to moderately increased signal intensity. Fibrous central scar is of very low signal intensity (arrowheads) as treatment for these patients varies depending on these factors. It is also important to document whether vascular invasion or distant metastasis is present.

\subsubsection{Fibrolamellar HCC}

Fibrolamellar HCC (FL-HCC) is a less aggressive tumor with a better prognosis than typical HCC. It consists of malignant hepatocytes separated into cords by fibrous strands. On CT, FL-HCC appears as a large, well-defined vascular mass with lobulated surface and often a central scar and calcifications in up to $70 \%$ of cases [64, 65]. On MR imaging, FL-HCC are typically hypointense on T1- and hyperintense on $\mathrm{T} 2$-weighted images, with the central scar being hypointense on both sequences (Fig. 17.16). This is in contrast to the scar of FNH, which is most often hyperintense on T2-weighted images. The fibrous central zones of both FNH and FL-HCC show delayed retention of CT and extracellular gadolinium MR contrast agents. By comparison with $\mathrm{FNH}$, the contrast enhancement in FL-HCC is usually heterogeneous compared with the often homogeneous contrast enhancement pattern of FNH.

\subsubsection{Cholangiocellular Carcinoma}

Cholangiocellular carcinoma (CCC) is the second most common primary malignancy of the liver. Intrahepatic CCC originates from the intralobular bile ducts (in contrast to hilar CCC, which arises from a main hepatic duct or from the bifurcation). Intrahepatic CCC often presents late as a large mass [66]. According to the growth characteristics, CCC is classified as mass forming, periductal infiltrating, or intra- ductal growing, with the mass-forming type being most common in intrahepatic CCC [66]. At CT and MR imaging, lesions tend to be hypodense at unenhanced $\mathrm{CT}$ and hypointense on T1-weighted images, with peripheral enhancement at dynamic contrast-enhanced studies [67]. Delayed phase CT/MR imaging (after 5-15 min) may show enhancement homogeneously or in the center of the lesion due to its rich fibrous stroma, which is suggestive of the diagnosis of CCC [68]. Interestingly, the central fibrotic stroma often shows signal suppression on diffusion-weighted MRI and return relatively high ADC value (Fig. 17.17). Periductal infiltrative CCC causes early segmental dilatation of bile ducts in a stage when the tumor itself may be difficult to discern [67]. In addition, there are morphologic features that can suggest the diagnosis of CCC. Peripheral lesions often demonstrate overlying capsular retraction due to their scirrhous, fibrous matrix (Fig. 17.18). Dilated intrahepatic bile ducts proximal to an intrahepatic CCC can also provide clues to the diagnosis, as biliary obstruction is usual with intrahepatic metastases (with the exception of colorectal cancer [69].

\subsection{Rare Primary Liver Tumors}

\subsubsection{Biliary Cystadenoma/ Cystadenocarcinomas}

These tumors present a similar appearance and morphology as their mucinous counterparts in the pancreas and occur usually in women. Even when benign, these tumors have a propensity for malignant degeneration, and any such tumor should be considered as potentially malignant. They appear as unilocular or multilocular cystic masses, with the typical anechoic and hypoechoic US appearance and near water-like attenuation 


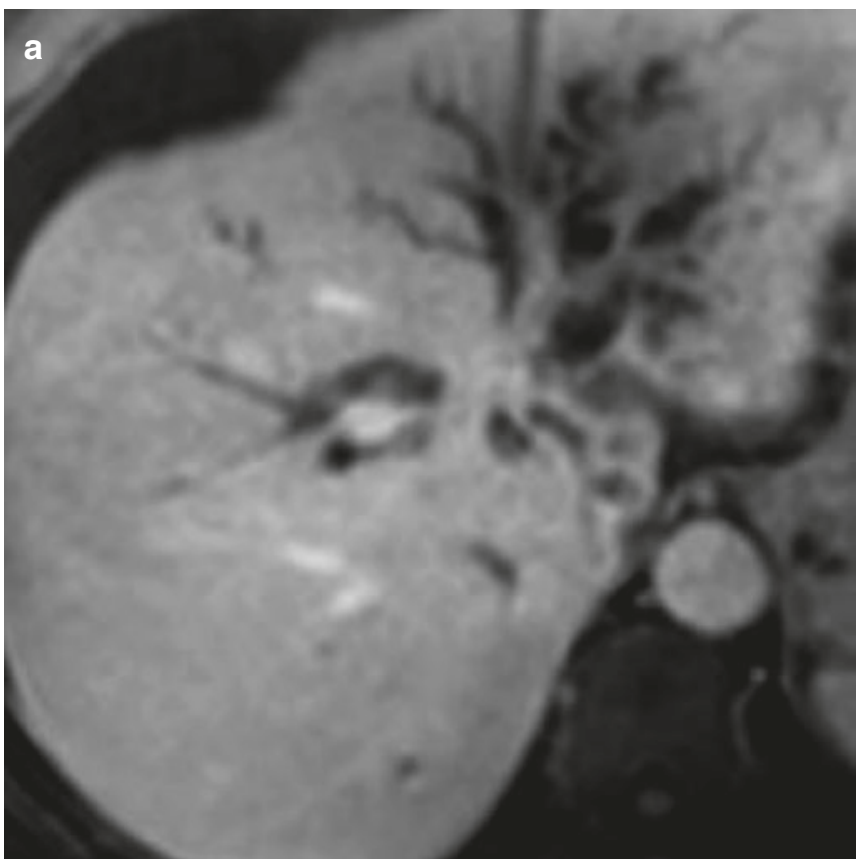

Fig. 17.17 Hilar cholangiocarcinoma: elderly man with progressive jaundice. (a) Contrast-enhanced T1-weighted image in the arterial phase shows dilatation of the intrahepatic ducts, which extend to the

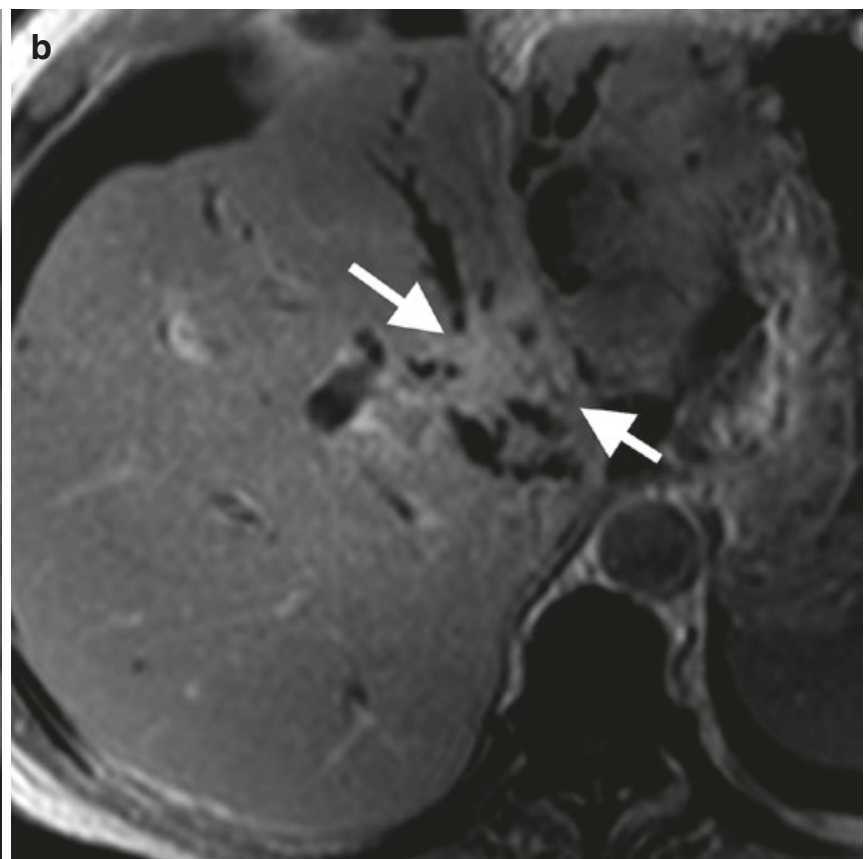

hepatic hilum. On the (b) 10 mins' delayed image, the tumor demonstrates late enhancement, which allows better delineation of the tumor (arrows) from the surrounding hepatic parenchyma
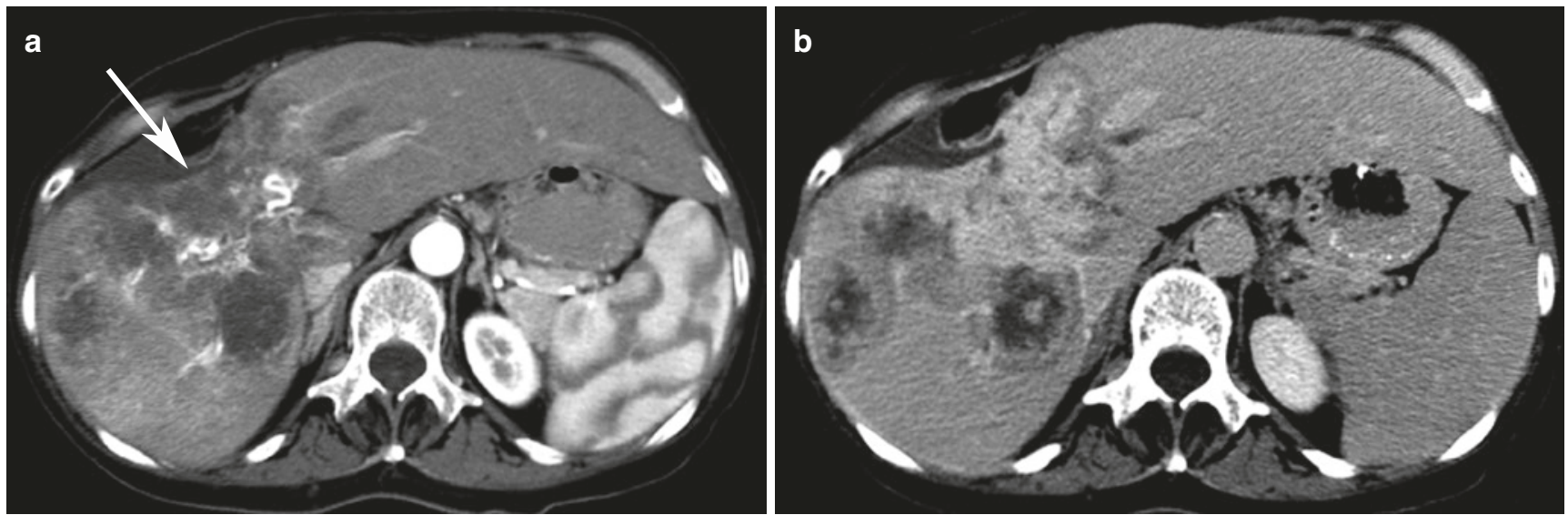

Fig. 17.18 Peripheral cholangiocarcinoma. (a) Contrast-enhanced CT in the arterial phase demonstrates a multicentric hypovascular mass with capsular retraction (arrow). (b) Delayed phase demonstrated typical late enhancement due to fibrous matrix

contents on CT, with peripheral soft tissue nodularity and traversing septations. The greater presence of papillary excrescences, soft tissue nodularity or septations, are associated with a higher risk of malignancy [70]. The cystic areas show variable signal intensity at T1-weighted MRI, including being hyperintense to liver related to its proteinaceous content. Coarse calcifications may be observed at US and CT in both cystadenoma and cystadenocarcinoma and is not a sign of benignity.

\subsubsection{Hepatic Angiosarcoma}

Hepatic angiosarcoma is a rare tumor. There is a strong association with prior exposure to carcinogens such as vinyl chloride and Thorotrast, as well as in patients with hemochromatosis. However, in the majority, the tumor is idiopathic. Pathologically, angiosarcoma presents as large, solitary masses or with multiple tumor nodules of varying size, which contain multiple vascular channels. 
The imaging appearance of angiosarcoma is often nonspecific, appearing hypodense on unenhanced CT, hypointense on T1-weighted MR imaging, and mildly hyperintense on T2-weighted imaging (although if prominent sinusoidal vascular spaces are present, these can appear of homogeneous and very high T2-weighted signal intensity). Following iodinated or gadolinium-based contrast administration, most lesions show nonspecific heterogeneous enhancement. Potentially problematic, however, are those tumors with prominent sinusoidal vascular spaces, because they can mimic the appearance of benign hemangioma on CT and MRI. The high MR T2-weighted signal in such lesions further compounds this problem. In most such cases, however, careful evaluation will show that the tumoral enhancement does not follow characteristics of blood pool at all phases or that there are other features, such as multiple lesions, that make the diagnosis of hemangioma unlikely $[71,72]$.

\subsubsection{Epithelioid Hemangioendothelioma}

Epithelioid hemangioendothelioma (EHE) is a rare tumor of vascular origin, not to be confused with infantile hemangioendothelioma, which is a very different tumor. These hepatic tumors are characterized by multiple, peripheral-based lesions that progressively become confluent masses. In addition to the unusual peripheral liver distribution, a key characteristic feature is the presence of overlying capsular retraction, due to the presence of fibrosis and scarring [73]. The CT attenuation or MR signal intensity characteristics are nonspecific, although occasional tumoral calcifications may be seen. Contrast enhancement with CT or MR gadolinium chelates often shows a central zone of decreased enhancement with marked peripheral enhancement (Fig. 17.19). The reverse pattern has also been observed with a central area of increased enhancement and peripheral decreased enhancement. Concentric zones of marked enhancement have also been reported. A visible branch of the portal or hepatic vein terminating at the periphery of these lesions t (lollipop sign) has also been described, although this is not pathognomonic of the disease [74]. Lesions often become confluent and may grow large enough to replace nearly the entire liver parenchyma.

\subsection{Hepatic Metastases}

At US, liver metastases can appear hypoechoic, isoechoic, or hyperechoic. On dynamic contrast-enhanced CT, most metastases appear hypovascular and hypodense relative to liver parenchyma on the portal venous phase (Fig. 17.20). Hypervascular metastases are most commonly seen in renal cell carcinoma, neuroendocrine tumors, sarcomas,

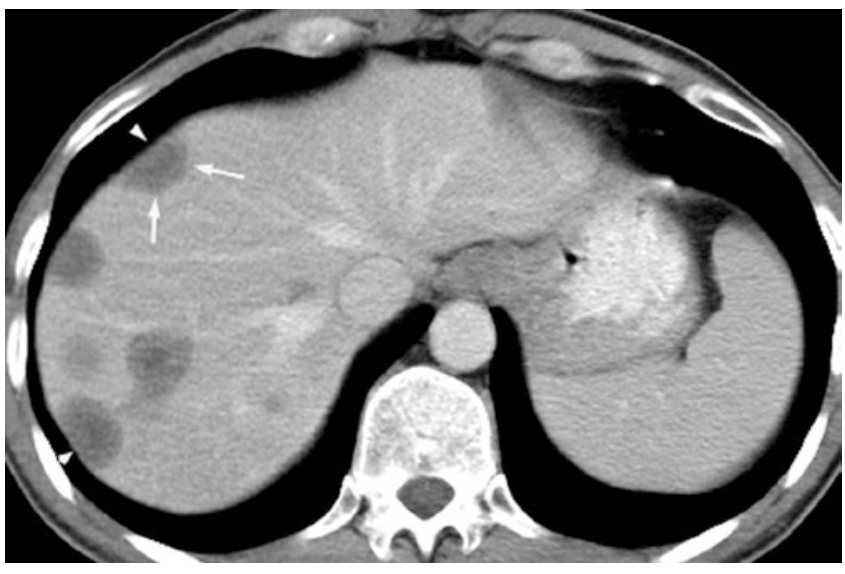

Fig. 17.19 Epithelioid hemangioendothelioma. Contrast CT (portal venous phase) shows multiple predominantly peripheral-based hypodense lesions. Note that some of the lesions show a laminated appearance (arrows). Early development of capsular retraction is present with flattening of the capsule overlying some of the lesions (arrowheads)

and breast tumor patients (Fig. 17.20). These tumors are best seen in the arterial phase and may become isodense and difficult to detect at the later phases of contrast enhancement. At MR, metastases are usually hypointense on T1-weighted and hyperintense on T2-weighted images [75]. Peritumoral edema makes lesions appear larger on T2-weighted images and is highly suggestive of a malignant mass [76]. High signal intensity on T1-weighted sequences is typical for melanoma metastases due to the paramagnetic nature of melanin. Some lesions may have a central area of hyperintensity (target sign) on T2-weighted images, which corresponds to central necrosis. DWI with high b-values (e.g., 600-800) is very helpful for detecting small liver metastases, which may otherwise escape detection (Fig. 17.21). On dynamic contrast-enhanced MR imaging, metastases demonstrate enhancement characteristics similar to those described for CT. Metastases may demonstrate a hypointense rim compared with the center of the lesion on delayed images (peripheral washout sign), which is highly specific for malignancy. It has been shown in colorectal cancer that the combination of using DWI, together with liver-specific contrast media, enhanced MRI results in the highest diagnostic accuracy for the detection of liver metastases (Fig. 17.22) [77].

\subsection{Differential Diagnosis of Focal Liver Lesions}

The approach to characterizing a focal liver lesion seen on CT begins with determining its density. If the lesion shows near water density, is homogenous in character, and has 

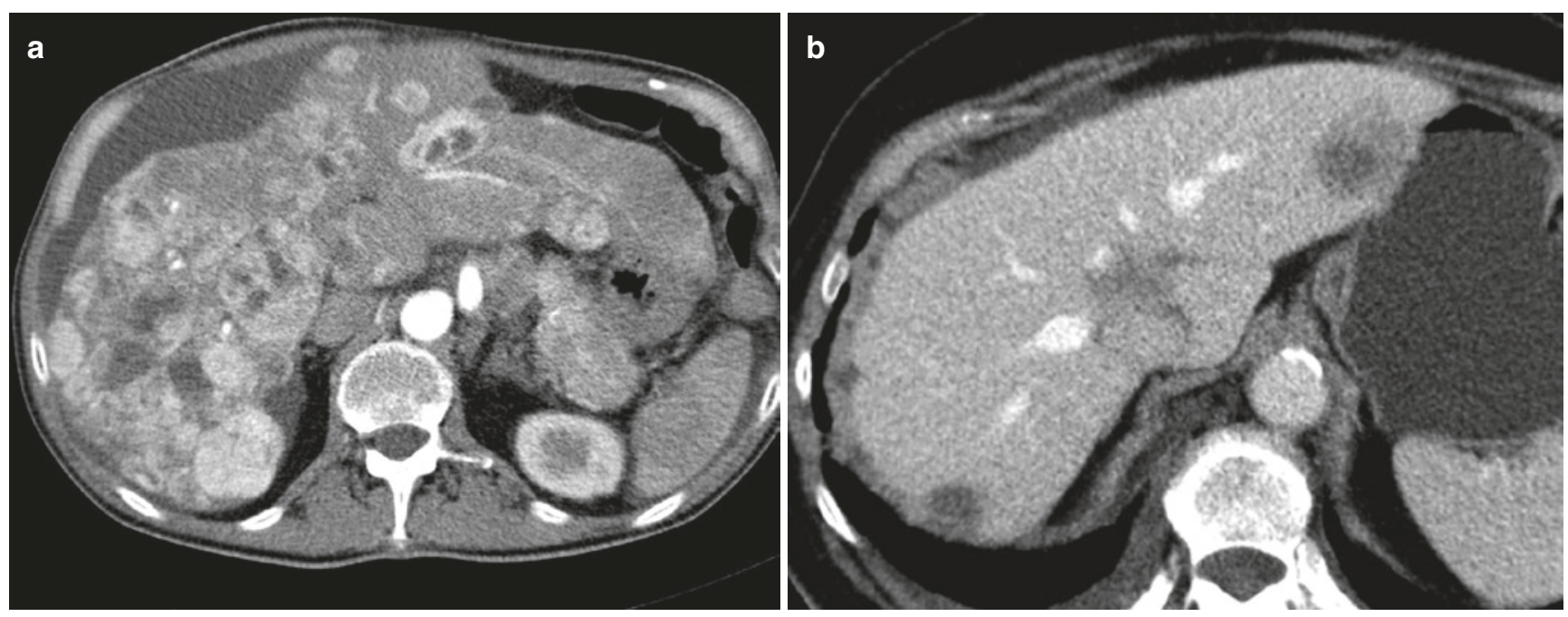

Fig. 17.20 Metastases. (a) Contrast-enhanced MDCT in the arterial phase demonstrates several predominantly hypervascular liver metastases of neuroendocrine cancer of the pancreas. (b) Contrast-enhanced MDCT in the venous phase shows typical hypovascular colorectal metastases

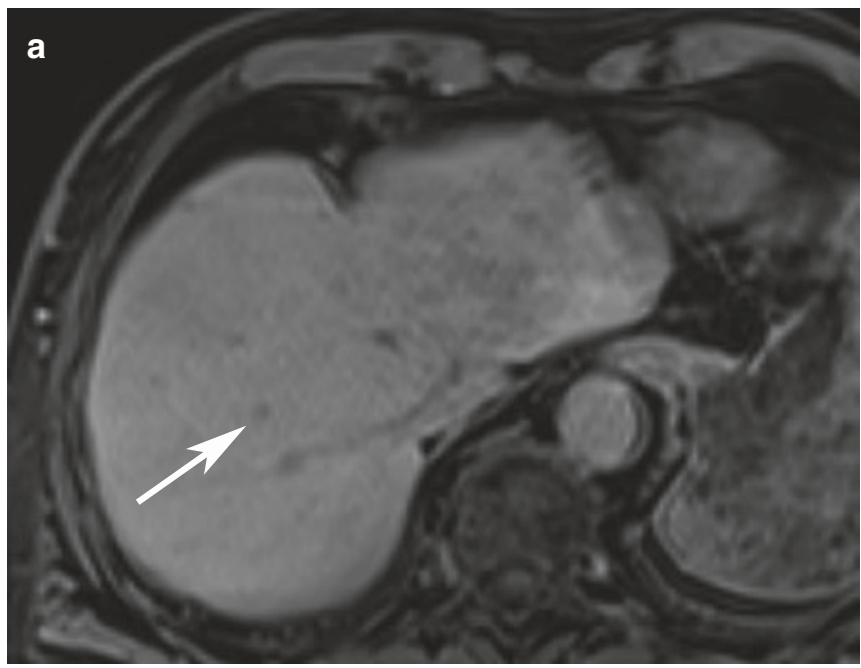

Fig. 17.21 Value of diffusion-weighted MRI for detection of small metastases. (a) Contrast-enhanced MRI shows one small metastasis in the right lobe (arrow). There is a subtle hypointensity in the right lobe

sharp margins, then a cyst should be considered and can be confirmed with US, equilibrium-phase CT, or even MR imaging (T2 bright and non-enhancing post-gadolinium), which can ensure there are no solid components or mural wall lesions. However, the radiologist should be familiar with the imaging features of other cystic lesions that can mimic simple cysts.

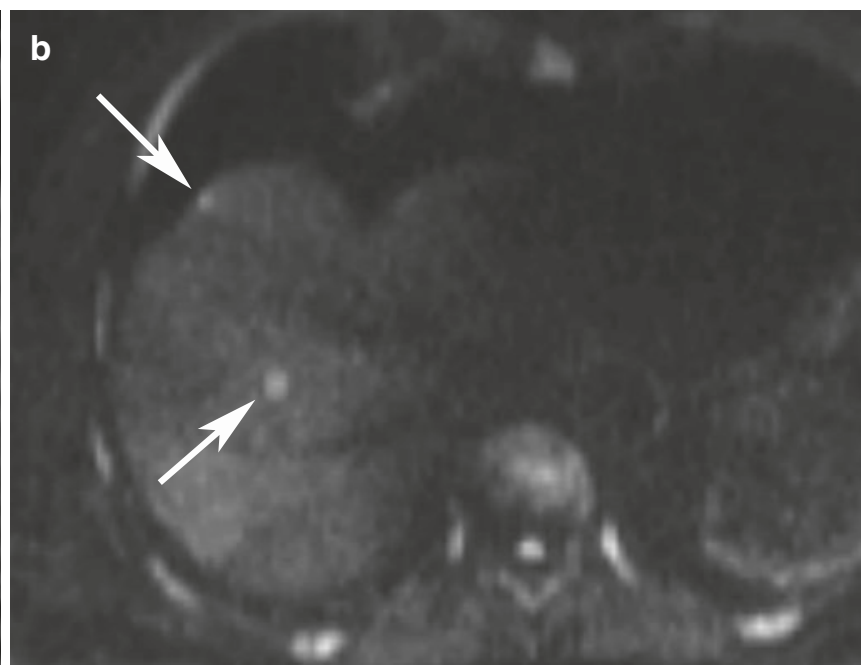

in a subcapsular location. (b) DWI clearly shows that there is an additional metastasis (arrows)

When evaluating solid focal liver lesions, disease characterization is largely reliant on observing the rate and pattern of contrast enhancement. If a lesion shows peripheral and nodular enhancement, with the density of enhancing portions showing the same general levels of blood vessels in the arterial, venous, and delayed phases, a hemangioma can be confidently diagnosed. Arterially hypervascular enhancing 

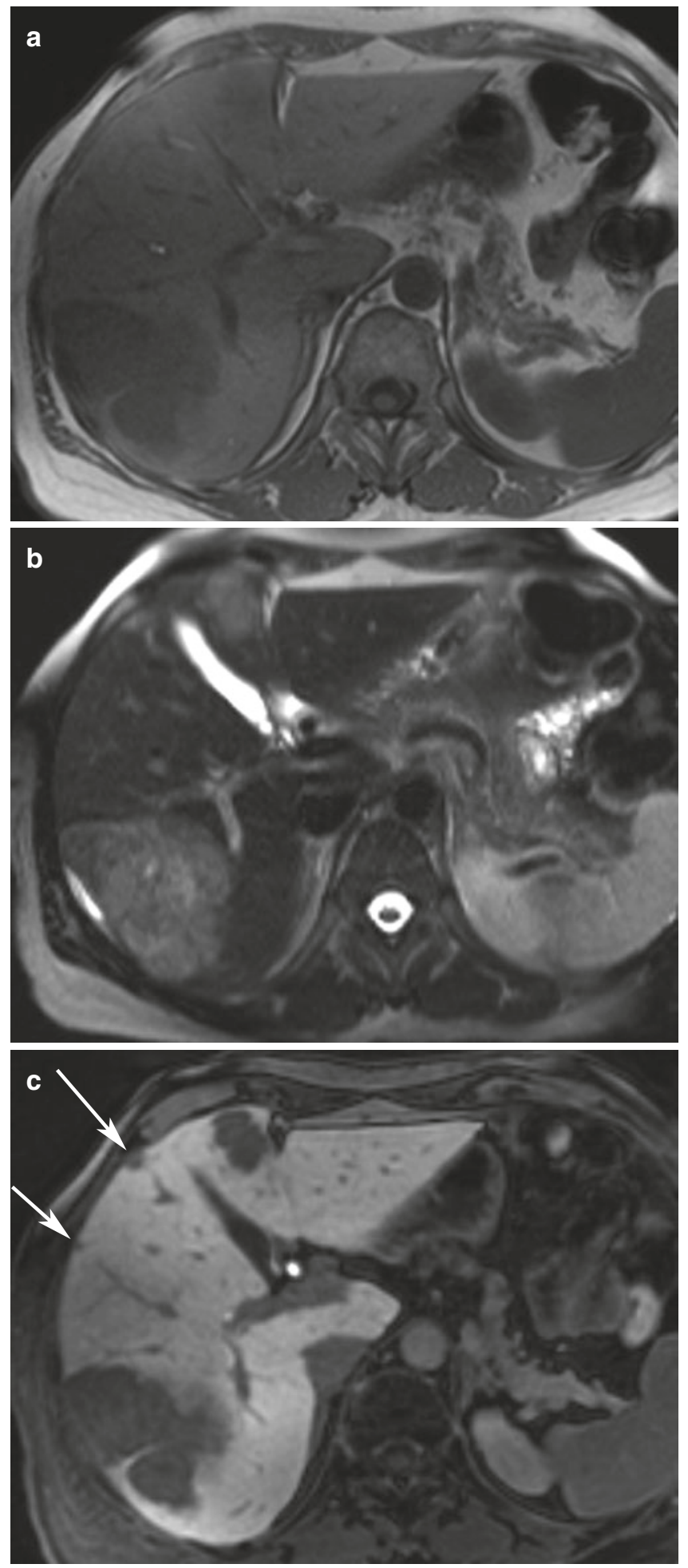

Fig.17.22 Colorectal liver metastases at gadoxetic acid-enhanced MRI. (a) Unenhanced T1-weighted MRI shows two hypointense lesions in segments 6/7 and 4. (b) The T2-weighted TSE image shows the lesions to be moderately hyperintense. (c) The gadoxetic-enhanced T1-weighted GRE image in the hepatobiliary phase shows two additional small subcapsular metastases (arrows) not seen on unenhanced MRI or MDCT (not shown) lesions include FNH, HCA, HCC, and metastases from neuroendocrine tumors, melanoma, renal cell carcinoma, and breast cancer. In general, HCC is considered in a setting of cirrhosis or chronic liver disease. FNH is most likely in young women with a non-cirrhotic liver and if the lesion is homogeneous and near-isodense/near-isointense on unenhanced CT/MR imaging with a central T2-weighted hyperintense scar.

By comparison, thick, irregular, heterogeneous enhancement or the presence of peripheral washout at the delayed phase suggests a malignant mass, such as metastases, CCC, or even HCC. In particular, delayed enhancement is a feature of $\mathrm{CC}$ due to is fibrotic stroma.

Liver-specific MR contrast has been shown to improve the characterization of FNH and HCA, increase the detection of suspicious focal lesions in patients with liver cirrhosis, as well as the identification of small focal liver lesions.

DWI is also now routinely performed in liver imaging. Its main clinical benefit is the detection of focal liver lesions, which may be missed on conventional and contrast-enhanced imaging sequences. Quantitative ADC measurements can support the characterization of focal liver lesions, with higher ADC values (e.g., $>1.7 \times 10-3 \mathrm{~mm}^{2} / \mathrm{s}$ ) favoring benign lesions [22]. However, the use of ADC value should be made with the knowledge of the scanner ADC repeatability, as well as in collaboration with all other imaging findings because of the significant overlap of ADC values between benign and malignant lesions.

\section{Take-Home Messages}

- Contrast-enhanced liver MDCT for detection and characterization of focal masses should be at least biphasic, with a quadruple-phasic protocol being recommended for HCC detection and characterization in cirrhotic patients.

- MRI protocol should routinely include dynamic contrast-enhanced pulse sequences and DWI.

- Liver-specific MR contrast agents are recommended for evaluation of patients with potentially resectable colorectal liver metastases.

- Liver-specific MR contrast agents are helpful for characterization of FNH and adenoma and may increase the reader confidence in HCC characterization. 


\section{References}

1. Laghi A. Multidetector CT (64 slices) of the liver: examination techniques. Eur Radiol. 2007;17:675-83.

2. Weg N, Scheer MR, Gabor MP. Liver lesions: improved detection with dual-detector-array $\mathrm{CT}$ and routine $2.5-\mathrm{mm}$ thin collimation. Radiology. 1998;209:417-26.

3. Ichikawa T, Nakajima H, Nanbu A, et al. Effect of injection rate of contrast material on CT of hepatocellular carcinoma. AJR Am J Roentgenol. 2006;186:1413-8.

4. Foley WD, Hoffmann RG, Quiroz FA, et al. Hepatic helical CT: contrast material injection protocol. Radiology. 1994;192:367-71.

5. Kim T, Murakami T, Takahashi S, et al. Effects of injection rates of contrast material on arterial phase hepatic CT. AJR Am J Roentgenol. 1998;171:429-32.

6. Schima W, Hammerstingl R, Catalano C, et al. Quadruple-phase MDCT of the liver in patients with suspected hepatocellular carcinoma: effect of contrast material flow rate. AJR Am J Roentgenol. 2006;186:1571-9.

7. Sultana S, Awai K, Nakayama Y, et al. Hypervascular hepatocellular carcinomas: bolus tracking with a 40-detector CT scanner to time arterial phase imaging. Radiology. 2007;243:140-7.

8. Oliver JH, Baron RL. Helical biphasic contrast-enhanced CT of the liver: technique, indications, interpretations and pitfalls. Radiology. 1996;201:1-14.

9. Vardhanabhuti V, Loader R, Roobottom CA. Assessment of image quality on effects of varying tube voltage and automatic tube current modulation with hybrid and pure iterative reconstruction techniques in abdominal/pelvic CT: a phantom study. Investig Radiol. 2013;48:167-74.

10. Singh S, Kalra M, Hsieh J, et al. Abdominal CT: comparison of adaptive statistical iterative and filtered back projection reconstruction techniques. Radiology. 2010;257:373-83.

11. May MS, Wüst W, Brand M, et al. Dose reduction in abdominal computed tomography: intraindividual comparison of image quality of full-dose standard and half-dose iterative reconstructions with dual-source computed tomography. Investig Radiol. 2011;46:465-70.

12. Gonzalez-Guindalini FD, Botelho MP, Töre HG, et al. MDCT of chest, abdomen, and pelvis using attenuation-based automated tube voltage selection in combination with iterative reconstruction: an intrapatient study of radiation dose and image quality. AJR Am J Roentgenol. 2013;201:1075-82.

13. Fuentes-Orrego JM, Hayano K, Kambadakone AR, et al. Dosemodified 256-MDCT of the abdomen using low tube current and hybrid iterative reconstruction. Acad Radiol. 2013;20:1405-12.

14. Altenbernd J, Heusner TA, Ringelstein A, Ladd SC, Forsting M, Antoch G. Dual-energy-CT of hypervascular liver lesions in patients with HCC: investigation of image quality and sensitivity. Eur Radiol. 2011;21:738-43.

15. Luo XF, Xie XQ, Cheng S, et al. Dual-energy CT for patients suspected of having liver iron overload: can virtual iron content imaging accurately quantify liver iron content? Radiology. 2015;277:95-103

16. Lee MH, Kim YK, Park MJ, Hwang J, Kim SH, Lee WJ, Choi D. Gadoxetic acid-enhanced fat suppressed three-dimensional T1-weighted MRI using a multiecho dixon technique at 3 tesla: emphasis on image quality and hepatocellular carcinoma detection. J Magn Reson Imaging. 2013;38:401-10.

17. Chandarana H, Block KT, Winfeld MJ, et al. Free-breathing contrast-enhanced T1-weighted gradient-echo imaging with radial k-space sampling for paediatric abdominopelvic MRI. Eur Radiol. $2014 ; 24: 320-6$
18. Padhani AR, Liu G, Chenevert TL, et al. Diffusion-weighted magnetic resonance imaging as a cancer biomarker: consensus and recommendations. Neoplasia. 2009;11:102-25.

19. Koh DM, Brown G, Riddell AM, et al. Detection of colorectal hepatic metastases using MnDPDP MR imaging and diffusionweighted imaging (DWI) alone and in combination. Eur Radiol. 2008;18:903-10.

20. Holzapfel K, Reiser-Erkan C, Fingerle AA, et al. Comparison of diffusion-weighted MR imaging and multidetector-row $\mathrm{CT}$ in the detection of liver metastases in patients operated for pancreatic cancer. Abdom Imaging. 2011;36:179-84.

21. Vandecaveye V, De Keyzer F, Verslype C, et al. Diffusion-weighted MRI provides additional value to conventional dynamic contrastenhanced MRI for detection of hepatocellular carcinoma. Eur Radiol. 2009; 19:2456-66.

22. Taouli B, Koh DM. Diffusion-weighted MR imaging of the liver. Radiology. 2010;254:47-66.

23. Oudkerk M, Torres CG, Song B, et al. Characterization of liver lesions with mangafodipir trisodium-enhanced MR imaging: multicenter study comparing MR and dual-phase spiral CT. Radiology. 2002;223:517-24.

24. Scharitzer M, Schima W, Schober E, et al. Characterization of hepatocellular tumors: value of mangafodipir-enhanced magnetic resonance imaging. J Comput Assist Tomogr. 2005;29:181-90.

25. Ward J, Robinson PJ, Guthrie JA, et al. Liver metastases in candidates for hepatic resection: comparison of helical CT and gadolinium- and SPIO-enhanced imaging. Radiology. 2005;237:170-80.

26. Hammerstingl R, Huppertz A, Breuer J, et al. Diagnostic efficacy of gadoxetic acid (Primovist)-enhanced MRI and spiral CT for a therapeutic strategy: comparison with intraoperative and histopathologic findings in focal liver lesions. Eur Radiol. 2008;18:457-67.

27. Schima W, Saini S, Echeverri JA, et al. T2-weighted MR imaging for characterization of focal liver lesions: conventional spin-echo vs fast spin-echo. Radiology. 1997;202:389-93.

28. Farraher SW, Jara H, Chang KJ, et al. Differentiation of hepatocellular carcinoma and hepatic metastasis from cysts and hemangiomas with calculated $\mathrm{T} 2$ relaxation times and the $\mathrm{T} 1 / \mathrm{T} 2$ relaxation times ratio. J Magn Reson Imaging. 2006;24:1333-41.

29. Semelka RC, Brown ED, Ascher SM, et al. Hepatic hemangiomas: a multi-institutional study of appearance on T2-weighted and serial gadolinium-enhanced gradient-echo MR images. Radiology. 1994;192:401-6.

30. Oto A, Kulkarni K, Nishikawa R, Baron RL. Contrast enhancement of hepatic hemangiomas on multiphase MDCT: can we diagnose hepatic hemangiomas by comparing enhancement with blood pool? AJR Am J Roentgenol. 2010;195:381-6.

31. Ba-Ssalamah A, Uffmann M, Saini S, et al. Clinical value of MRI liver-specific contrast agents: a tailored examination for a confident noninvasive diagnosis of focal liver lesions. Eur Radiol. 2009;19:342-57.

32. Vossen JA, Buijs M, Liapi E, et al. Receiver operating characteristic analysis of diffusion-weighted magnetic resonance imaging in differentiating hepatic hemangioma from other hypervascular liver lesions. J Comput Assist Tomogr. 2008;32:750-6.

33. Kehagias D, Moulopoulos L, Antoniou A, et al. Focal nodular hyperplasia: imaging findings. Eur Radiol. 2001;11:202-12.

34. Brancatelli G, Federle MP, Grazioli L, et al. Focal nodular hyperplasia: CT findings with emphasis on multiphasic helical CT in 78 patients. Radiology. 2001;219:61-8.

35. Uggowitzer MM, Kugler C, Mischinger HJ, et al. Echo-enhanced Doppler sonography of focal nodular hyperplasia of the liver. $\mathrm{J}$ Ultrasound Med. 1999;18:445-51.

36. McInnes MD, Hibbert RM, Inácio JR, Schieda N. Focal nodular hyperplasia and hepatocellular adenoma: accuracy of gadoxetic 
acid-enhanced MR imaging — a systematic review. Radiology. 2015;277:413-23.

37. Purysko AS, Remer EM, Coppa CP, et al. Characteristics and distinguishing features of hepatocellular adenoma and focal nodular hyperplasia on gadoxetate disodium-enhanced MRI. AJR Am J Roentgenol. 2012;198:115-23.

38. Leconte I, Van Beers BE, Lacrosse M, et al. Focal nodular hyperplasia: natural course observed with CT and MRI. J Comput Assist Tomogr. 2000;24:61-6.

39. Mathieu D, Kobeiter H, Maison P, et al. Oral contraceptive use and focal nodular hyperplasia of the liver. Gastroenterology. 2000;118:560-4.

40. Prasad SR, Sahani DV, Mino-Kenudson M, et al. Benign hepatic neoplasms: an update on cross-sectional imaging spectrum. J Comput Assist Tomogr. 2008;32:829-40.

41. Katabathina VS, Menias CO, Shanbhogue AK, et al. Genetics and imaging of hepatocellular adenomas: 2011 update. Radiographics. 2011;31:1529-43.

42. van Aalten SM, Thomeer MG, Terkivatan T, et al. Hepatocellular adenomas: correlation of MR imaging findings with pathologic subtype classification. Radiology. 2011;261:172-81.

43. Bioulac-Sage P, Sempoux C, Balabaud C. Hepatocellular adenoma: classification, variants and clinical relevance. Semin Diagn Pathol. 2017;34:112-25.

44. Nault JC, Paradis V, Cherqui D, Vilgrain V, Zucman-Rossi J. Molecular classification of hepatocellular adenoma in clinical practice. J Hepatol. 2017;67:1074-83.

45. Semelka RC, Hussain SM, Marcos HB, Woosley JT. Biliary hamartomas: solitary and multiple lesions shown on current MR techniques including gadolinium enhancement. J Magn Reson Imaging. 1999;10:196-201.

46. Martin DR, Kalb B, Sarmiento JM, et al. Giant and complicated variants of cystic bile duct hamartomas of the liver: MRI findings and pathological correlations. J Magn Reson Imaging. 2010;31:903-11.

47. Jeffrey RB Jr, Tolentino CS, Chang FC, Federle MP. CT of small pyogenic hepatic abscesses: the cluster sign. AJR Am J Roentgenol. 1988;151:487-9.

48. Barreda R, Ros PR. Diagnostic imaging of liver abscess. Crit Rev Diagn Imaging. 1992;33:29-58.

49. Laghi A, Iannaccone R, Rossi P, et al. Hepatocellular carcinoma: detection with triple-phase multi-detector row helical CT in patients with chronic hepatitis. Radiology. 2003;226:543-9.

50. Ichikawa T, Kitamura T, Nakajima H, et al. Hypervascular hepatocellular carcinoma: can double arterial phase imaging with multidetector CT improve tumor depiction in the cirrhotic liver? AJR Am J Roentgenol. 2002;179:751-8.

51. Monzawa S, Ichikawa T, Nakajima H, et al. Dynamic CT for detecting small hepatocellular carcinoma: usefulness of delayed phase imaging. AJR Am J Roentgenol. 2007;188:147-53.

52. Iannacone R, Laghi A, Catalano C, et al. Hepatocellular carcinoma: role of unenhanced and delayed-phase multi-detector row helical CT in patients with cirrhosis. Radiology. 2005;234:460-7.

53. Baron RL, Brancatelli G. Computed tomographic imaging of hepatocellular carcinoma. Gastroenterology. 2004;127:S133-43.

54. Forner A, Vilana R, Ayuso C, et al. Diagnosis of hepatic nodules $20 \mathrm{~mm}$ or smaller in cirrhosis: prospective validation of the noninvasive diagnostic criteria for hepatocellular carcinoma. Hepatology. 2008:47:97-104.

55. Tublin ME, Dodd GD, Baron RL. Benign and malignant portal vein thrombosis: differentiation by CT characteristics. AJR Am J Roentgenol. 1997;168:719-23.

56. Stevens WR, Gulino SP, Batts KP, et al. Mosaic pattern of hepatocellular carcinoma: histologic basis for a characteristic CT appearance. J Comput Assist Tomogr. 1996;20:337-42.
57. Choi JW, Lee JM, Kim SJ, et al. Hepatocellular carcinoma: imaging patterns on gadoxetic acid-enhanced MR images and their value as an imaging biomarker. Radiology. 2013;267:776-86.

58. Chen L, Zhang L, Bao J, et al. Comparison of MRI with liverspecific contrast agents and multidetector row $\mathrm{CT}$ for the detection of hepatocellular carcinoma: a meta-analysis of 15 direct comparative studies. Gut. 2013;62:1520-1.

59. Song KD, Kim SH, Lim HK, Jung SH, Sohn I, Kim HS. Subcentimeter hypervascular nodule with typical imaging findings of hepatocellular carcinoma in patients with history of hepatocellular carcinoma: natural course on serial gadoxetic acid-enhanced MRI and diffusionweighted imaging. Eur Radiol. 2015;25:2789-96.

60. Kim TK, Lee KH, Jang JJ, et al. Analysis of gadobenate dimeglumine-enhanced MR findings for characterizing small (1-2$\mathrm{cm}$ ) hepatic nodules in patients at high risk for hepatocellular carcinoma. Radiology. 2011;259:730-8.

61. Lee MH, Kim SH, Park MJ, et al. Gadoxetic acid-enhanced hepatobiliary phase MRI and high-b-value diffusion-weighted imaging to distinguish well-differentiated hepatocellular carcinomas from benign nodules in patients with chronic liver disease. AJR Am J Roentgenol. 2011;197:W868-75.

62. Bruix J, Sherman M. Management of hepatocellular carcinoma: an update. Hepatology. 2011;53:1020-2.

63. McEvoy SH, McCarthy CJ, Lavelle LP, et al. Hepatocellular carcinoma: illustrated guide to systematic radiologic diagnosis and staging according to guidelines of the American Association for the Study of Liver Diseases. Radiographics. 2013;33:1653-68.

64. Ichikawa T, Federle MP, Grazioli L, et al. Fibrolamellar hepatocellular carcinoma: imaging and pathologic findings in 31 recent cases. Radiology. 1999;213:352-61.

65. Ichikawa T, Federle MP, Grazioli L, Marsh W. Fibrolamellar hepatocellular carcinoma: pre- and posttherapy evaluation with CT and MR imaging. Radiology. 2000;217:145-51.

66. Lim JH. Cholangiocarcinoma: morphologic classification according to growth pattern and imaging findings. AJR Am J Roentgenol. 2003;181:819-27.

67. Han JK, Choi BI, Kim AY, et al. Cholangiocarcinoma: pictorial essay of CT and cholangiographic findings. Radiographics. 2002;22:173-87.

68. Lee WJ, Lim HK, Jang KM, et al. Radiologic spectrum of cholangiocarcinoma: emphasis on unusual manifestations and differential diagnoses. Radiographics. 2001;21:S97-S116.

69. Jhaveri KS, Halankar J, Aguirre D, et al. Intrahepatic bile duct dilatation due to liver metastases from colorectal carcinoma. AJR Am J Roentgenol. 2009;193:752-6.

70. Buetow PC, Buck JL, Pantongrag-Brown L, et al. Biliary cystadenoma and cystadenocarcinoma: clinical-imaging pathologic correlations with emphasis on the importance of ovarian stroma. Radiology. 1995;196:805-10.

71. Peterson MS, Baron RL, Rankin SC. Hepatic angiosarcoma: findings on multiphasic contrast-enhanced helical CT do not mimic hepatic hemangioma. AJR Am J Roentgenol. 2000; $175: 165-70$.

72. Koyama T, Fletcher JG, Johnson CD, et al. Primary hepatic angiosarcoma: findings at CT and MR imaging. Radiology. 2002;222:667-73.

73. Miller WJ, Dodd GD 3rd, Federle MP, Baron RL. Epithelioid hemangioendothelioma of the liver: imaging findings with pathologic correlation. AJR Am J Roentgenol. 1992;159:53-7.

74. Alomari AI. The lollipop sign: a new cross-sectional sign of hepatic epithelioid hemangioendothelioma. Eur J Radiol. 2006;59:460-4. 
75. Schima W, Kulinna C, Langenberger H, Ba-Ssalamah A. Liver metastases of colorectal cancer: US, CT or MR? Cancer Imaging. 2005;5:S149-56.

76. Lee MJ, Saini S, Compton CC, Malt RA. MR demonstration of edema adjacent to a liver metastasis: pathologic correlation. AJR Am J Roentgenol. 1991;157:499-501.
77. Vilgrain V, Esvan M, Ronot M, Caumont-Prim A, Aubé C, Chatellier G. A meta-analysis of diffusion-weighted and gadoxetic acid-enhanced MR imaging for the detection of liver metastases. Eur Radiol. 2016;26:4595-615.

Open Access This chapter is licensed under the terms of the Creative Commons Attribution 4.0 International License (http://creativecommons. org/licenses/by/4.0/), which permits use, sharing, adaptation, distribution and reproduction in any medium or format, as long as you give appropriate credit to the original author(s) and the source, provide a link to the Creative Commons license and indicate if changes were made.

The images or other third party material in this book are included in the book's Creative Commons license, unless indicated otherwise in a credit line to the material. If material is not included in the book's Creative Commons license and your intended use is not permitted by statutory regulation or exceeds the permitted use, you will need to obtain permission directly from the copyright holder. 